\title{
Clinical Management of Adult Patients with COVID- 19 Outside Intensive Care Units: Guidelines from the Italian Society of Anti-Infective Therapy (SITA) and the Italian Society of Pulmonology (SIP)
}

\author{
Matteo Bassetti (D) - Daniele Roberto Giacobbe (D) - Paolo Bruzzi - Emanuela Barisione $\cdot$ Stefano Centanni • \\ Nadia Castaldo · Silvia Corcione • Francesco Giuseppe De Rosa • Fabiano Di Marco • Andrea Gori • \\ Andrea Gramegna · Guido Granata • Angelo Gratarola • Alberto Enrico Maraolo • Malgorzata Mikulska • \\ Andrea Lombardi · Federico Pea $\cdot$ Nicola Petrosillo $\cdot$ Dejan Radovanovic $\cdot$ Pierachille Santus · Alessio Signori · \\ Emanuela Sozio · Elena Tagliabue - Carlo Tascini · Carlo Vancheri • Antonio Vena • Pierluigi Viale • \\ Francesco Blasi on behalf of the Italian Society of Anti-infective Therapy (SITA) and the Italian Society of \\ Pulmonology (SIP)
}

Received: May 27, 2021 / Accepted: June 15, 2021 / Published online: July 30, 2021

(C) The Author(s) 2021

\section{ABSTRACT}

Introduction: The Italian Society of Anti-Infective Therapy (SITA) and the Italian Society of Pulmonology (SIP) constituted an expert panel for developing evidence-based guidance for the

The Italian Society of Anti-infective Therapy (SITA) and the Italian Society of Pulmonology (SIP) Collaborators members are listed in the Acknowledgements section.

Supplementary Information The online version contains supplementary material available at https:// doi.org/10.1007/s40121-021-00487-7.

M. Bassetti ( $₫) \cdot$ D. R. Giacobbe $(\bowtie) \cdot$ M. Mikulska • A. Vena

Infectious Diseases Unit, Ospedale Policlinico San Martino-IRCCS, L.go R. Benzi, 10, 16132 Genoa, Italy e-mail: matteo.bassetti@unige.it

D. R. Giacobbe

e-mail: danieleroberto.giacobbe@unige.it

M. Bassetti - D. R. Giacobbe · M. Mikulska Department of Health Sciences (DISSAL), University of Genoa, Genoa, Italy

P. Bruzzi

Clinical Epidemiology Unit, Ospedale Policlinico

San Martino-IRCCS, Genoa, Italy clinical management of adult patients with coronavirus disease 2019 (COVID-19) outside intensive care units.

Methods: Ten systematic literature searches were performed to answer ten different key questions. The retrieved evidence was graded according to the Grading of Recommendations Assessment, Development, and Evaluation methodology (GRADE).

Results and Conclusion: The literature searches mostly assessed the available evidence on the management of COVID-19 patients in terms

E. Barisione · E. Tagliabue

Interventional Pulmonology, Ospedale Policlinico San Martino-IRCCS, Genoa, Italy

\section{S. Centanni}

Department of Health Sciences, University of Milan, Respiratory Unit, ASST Santi Paolo e Carlo, Milan, Italy

N. Castaldo $\cdot$ E. Sozio $\cdot$ C. Tascini

Infectious Diseases Clinic, Santa Maria Misericordia Hospital, Udine, Italy

\section{S. Corcione - F. G. De Rosa}

Department of Medical Sciences, Infectious

Diseases, University of Turin, Turin, Italy 
of antiviral, anticoagulant, anti-inflammatory, immunomodulatory, and continuous positive airway pressure (CPAP)/non-invasive ventilation (NIV) treatment. Most evidence was deemed as of low certainty, and in some cases, recommendations could not be developed according to the GRADE system (best practice recommendations were provided in similar situations). The use of neutralizing monoclonal antibodies may be considered for outpatients at risk of disease progression. For inpatients, favorable recommendations were provided for anticoagulant prophylaxis and systemic steroids administration, although with low certainty of evidence. Favorable recommendations, with very low/low certainty of evidence, were also provided for, in specific situations, remdesivir, alone or in combination with baricitinib, and tocilizumab. The presence of many best practice recommendations testified to the need for further investigations by means of randomized controlled trials, whenever possible, with some possible future research directions stemming from the results of the ten systematic reviews.

S. Corcione

Tufts University School of Medicine, Boston, MA, USA

\section{F. Di Marco}

Department of Health Sciences, University of Milan, Respiratory Unit, ASST Papa Giovanni XXIII

Hospital, Bergamo, Italy

A. Gori · A. Gramegna · A. Lombardi · F. Blasi Department of Pathophysiology and

Transplantation, Università degli Studi di Milano,

Milan, Italy

A. Gori · A. Lombardi

Infectious Diseases Unit, Fondazione IRCCS $\mathrm{Ca}^{\prime}$

Granda Ospedale Maggiore Policlinico, Milan, Italy

A. Gramegna · F. Blasi

Fondazione IRCCS Ca' Granda Ospedale Maggiore

Policlinico, Internal Medicine Department,

Respiratory Unit and Cystic Fibrosis Adult Center,

Milan, Italy

A. Gori

Centre for Multidisciplinary Research in Health

Science (MACH), University of Milan, Milan, Italy

G. Granata · N. Petrosillo

Clinical and Research Department for Infectious

Diseases, National Institute for Infectious Diseases L.

Spallanzani, IRCCS, Rome, Italy
Keywords: COVID-19; SARS-CoV-2; Guidelines

\section{Key Summary Points}

The use of neutralizing monoclonal antibodies may be considered for outpatients at risk of disease progression.

For inpatients, favorable recommendations are provided for anticoagulant prophylaxis and systemic steroids administration, although with low certainty of evidence.

Favorable recommendations, with very low/low certainty of evidence, are also provided for, in specific situations, remdesivir, alone or in combination with baricitinib, and tocilizumab.

The presence of many best practice recommendations testifies to the need for further investigations by means of randomized controlled trials.

A. Gratarola

Department of Emergency and Urgency, San

Martino Policlinico Hospital, IRCCS, Genoa, Italy

A. E. Maraolo

First Division of Infectious Diseases, Cotugno

Hospital, AORN dei Colli, Naples, Italy

F. Pea $\cdot$ P. Viale

Department of Medical and Surgical Sciences, Alma

Mater Studiorum-University of Bologna, Bologna, Italy

F. Pea

SSD Clinical Pharmacology Unit, University

Hospital, IRCCS Azienda Ospedaliero Universitaria di Bologna, Bologna, Italy

N. Petrosillo

Infection Control and Infectious Disease Service, University Hospital "Campus-Biomedico", Rome, Italy

D. Radovanovic · P. Santus

Division of Respiratory Diseases, Ospedale L. Sacco, ASST Fatebenefratelli-Sacco, Milan, Italy

P. Santus

Department of Biomedical and Clinical Sciences (DIBIC), Università degli Studi di Milano, Milan, Italy 


\section{INTRODUCTION}

Coronavirus disease 2019 (COVID-19), caused by the severe acute respiratory syndrome coronavirus 2 (SARS-CoV-2), has affected and continues to affect millions of people worldwide $[1,2]$. Although most of infected individuals do not develop severe disease, the absolute numbers of those requiring hospital support during exponential increases of the virus diffusion have put extraordinary pressure on hospitals in Italy and in other European countries.

The rapid diffusion of this novel virus initially implied the lack of high-level evidence on any possible best therapeutic approach for both outpatients and inpatients with COVID-19. As a consequence, during the first months of the pandemic, the management of patients with COVID-19 was largely based on off-label and compassionate drug uses [3-5]. However, this can no longer be justified (at least on a large scale and outside specific situations) now that the unprecedented efforts of scientific research worldwide have produced (in just 1 year) many clinical studies, both observational and experimental, including randomized controlled trials (RCTs), that could allow for a more solid evidence-based therapeutic approach to patients with COVID-19. Of course, several gray areas remain, and further future refinement of current approaches is still a critical priority. However, this does not cancel the need to provide clinicians with an updated summary of the already available evidence for their reference, also considering the large amount of novel

A. Signori

Department of Health Sciences, Section of

Biostatistics, University of Genoa, Genoa, Italy

C. Vancheri

Regional Referral Centre for Rare Lung Diseases-

University Hospital "Policlinico G. Rodolico",

Catania, Italy

C. Vancheri

Department of Clinical and Experimental Medicine, University of Catania, Catania, Italy

P. Viale

Infectious Diseases Unit, University Hospital IRCCS

Policlinico Sant'Orsola, Bologna, Italy scientific literature on COVID-19 released every day. This information overload could also have the unintended effect of confounding clinicians if not inserted in a correct framework of certainty and applicability of the evidence.

Against this background, structured guidance through guidelines development is a fundamental step that needs to be taken to optimize the management of COVID-19 in Italy, which was one of the first and most affected countries in Europe. For this reason, the Italian Society of Anti-Infective Therapy (SITA) and the Italian Society of Pulmonology (SIP) jointly developed the current guidelines for the therapeutic management of patients with COVID-19. The current document is relevant to patients not requiring (or still not requiring) admission to intensive care unit (ICU).

\section{METHODS}

We set up a panel of experts from SITA and SIP, including members (D.R.G., P.B.) with previous experience in the development of documents and recommendations with the Grading of Recommendations Assessment, Development, and Evaluation (GRADE) system [6-8]. The aim was to address ten different key questions regarding the clinical management of adult outpatients and inpatients with COVID-19 outside ICU. The complete panel composition and roles are detailed in the Supplementary Material. The panel comprised infectious diseases specialists and pneumologists, reflecting the infectious nature of the disease and its frequent presentation as a respiratory syndrome. Furthermore, an intensive care physician (A.G.) and a pharmacologist (F.P.) were included in the panel to guarantee non-overlapping with intensive care management (delimitation of the target population of patients with COVID-19 outside ICU) and the point of view of a pharmacokinetic specialist in the panel, respectively.

The present guidelines mainly deal with administration of therapeutic agents to patients with COVID-19 and are targeted to physicians dealing with COVID-19 outside ICU, in order to provide a detailed appraisal of evidence and recommendations to support clinical practice. 
Given the absence of such a baseline appraisal, other associations/societies of physicians and patients were not involved in the development of the present first version of the guidelines. However, based on the certainty of evidence and the strength of recommendations provided by the present assessment, the possibility for either physicians' or patients' associations of suggesting preferences and critical areas needing further assessment/review will be provided through a structured questionnaire that will be made available on the websites of the two societies before the future pre-defined updates of the guidelines (see below).

The guidelines development process started with ten different systematic reviews of the literature, one for each question. The systematic reviews protocol followed the Preferred Reporting Items for Systematic Reviews and MetaAnalyses (PRISMA) statement [9]. For each systematic review, the inclusion criteria relied on the PICO strategy $(\mathrm{P}=$ population, $\mathrm{I}=$ intervention, $\mathrm{C}=$ comparison, $\mathrm{O}=$ outcomes). Specific P, I, C, and $\mathrm{O}$ were defined for each single question, as motivated in the discussion of evidence for the different questions (see below the dedicated evidence summaries for each question). The literature search was performed through PubMed, Embase, and the Cochrane COVID-19 Study Register. Specific search strings and flowcharts of the studies inclusion process for the ten different systematic searches are available in the Supplementary Material. The initial search period was from inception of January 2020 to 30 November 2020 , with two subsequent updates to 31 January 2021 and 30 April 2021. In line with the increasing availability of evidence from randomized studies over time, after 31 January 2021 updates of the guidelines text were limited to RCTs (after having verified that retrieved observational studies did not provide sufficient evidence to change already developed recommendations), including RCTs retrieved as not peer-reviewed pre-print manuscripts or press releases if deemed as potentially impacting recommendations. A further update of the literature search will be performed in November 2021. However, any potential practice-changing evidence that may become available before
November 2021 will be critically reviewed by the panel of experts in charge of preparing and reviewing the present guidelines, and, in case, incorporated into the recommendations. Any update will be published on the websites of the two societies (https://www.sitaonline.net and http://www.sipirs.it). Through the websites, patients' and physicians' societies will also be allowed to send their comments regarding areas needing further assessment/revision; that information will be taken into account and published alongside future versions of the guidelines.

For each question, recommendations were drafted by non-voting members based on the retrieved literature. The supporting literature for each recommendation was assessed independently using the GRADE system by D.R.G. and P.B., with disagreements being resolved by A.E.M. GRADE tables for each recommendation, available in the Supplementary Material, were prepared by evaluating the risk of bias, inconsistency, indirectness, and imprecision of the retrieved literature. For observational studies, the risk of bias was assessed by means of the Newcastle-Ottawa Scale (NOS) [10], whereas for RCTs the risk of bias was assessed by means of the Effective Practice and Organization of Care guidelines [11]. High risk of bias translated to "very serious risk of bias", low risk of bias translated to "no serious risk of bias", whereas moderate/unclear risk of bias translated to "serious risk of bias" or "no serious risk of bias" according to the evaluators' judgment. The certainty of evidence was graded as high, moderate, low, or very low. When sufficient evidence was not retrieved to allow the use of the GRADE system, recommendations were based on panel consensus, and defined as best practice recommendations.

A dedicated voting process (collection of voting forms through individual email messages) was developed for the present guidelines owing to the emergency situation and the clinical duties related to COVID-19, which eventually did not allow the organization of online meetings with participation of the full voting panel. More specifically, voting panel members were provided with the results of the various literature searches, the evidence 
summaries, the proposed recommendations, and the related GRADE tables. Each voting member was then allowed to individually vote (using a structured voting form) in favor or against each of the recommendations, to propose possible modifications, and to judge each recommendation as strong or weak according to GRADE rules [8]. For recommendations with an agreement $<75 \%$, further voting rounds were conducted after implementation of dedicated amendments based on the provided comments. After reaching an agreement $\geq 75 \%$ for all recommendations, all the authors reviewed and approved the final manuscript and Supplementary Material.

We recognize potential barriers to the implementation of the present guidelines, based on the availability/costs of therapeutic agents and possible off-label indications (e.g., for anti-inflammatory/immunomodulatory agents), that will continue to require standard local procedures for off-label administration, independent of the present guidelines. However, we also feel this will help to identify specific areas of further research (as detailed below for each question) that, in turn, may guide the design of further studies for increasing/revising the certainty of the evidence supporting the present recommendations. Finally, a survey will be developed and circulated among SITA and SIP members (and any other physicians' association requiring it through the societies websites) within October 2021 for evaluating implementation and adherence to the recommendations (through descriptive statistics and the related measures of uncertainty) and for collecting information about the barriers encountered to guidelines implementations. The results of the survey will be included in future guidelines versions.

This article is based on previously conducted studies and does not contain any new studies with human participants or animals performed by any of the authors.

\section{QUESTIONS ADDRESSED AND RECOMMENDATIONS}

A summary of questions and recommendations is provided in Table 1 , whereas a discussion of the retrieved evidence for each question is presented in the following paragraphs. Extended evidence summaries for questions 2 to 9 (i.e., those questions for which more details beyond those provided in the following paragraphs were deemed necessary for a complete presentation of evidence) are available in the Supplementary Material.

\section{Question 1: When Should a Patient with COVID-19 be Hospitalized?}

\section{Evidence Summary}

The need for hospital admission is an essential component of the initial evaluation of all patients with COVID-19 presenting at the emergency department or receiving home visits by family doctors. There is no general consensus on the optimal hospitalization criteria (either in general or in specific subgroups), and on how they should be applied in daily clinical practice [12-21].

Factors such as older age, male sex, presence of comorbidities, severe obesity, and shortness of breath have been consistently associated with hospitalization (as endpoint) in patients with COVID-19 across observational studies [12-28]. These factors likely reflect two different, nonmutually exclusive components: (1) an increased risk to progress to moderate/severe disease (e.g., comorbidities, male sex, severe obesity); (2) the presence of an already severe/ advanced disease (e.g., shortness of breath). Independent of what they reflect, the prognostic impact of these factors is the key determinant, since the major interest is to understand which patients may be safely followed/cured at home without a consequent, unfavorable prognostic effect. Nonetheless, this is seldom assessed directly, for two possible, different reasons: (1) lack of follow-up in non-hospitalized patients; (2) the endpoint is correctly prognostic (e.g., mortality, admission to ICU, recovery or improvement of clinical status), but 
Table 1 Summary of questions and recommendations

\section{Question Recommendations}

Question 1 When should a patient with COVID-19 be hospitalized?

Pending further evidence, it might be prudent not to base the decision to hospitalize or not patients with COVID-19 only on prognostic scores-weak recommendation, very low certainty of evidence

Hospitalization should be considered in patients with at least one of the following: low oxygen saturation on room air $\leq 92 \%$ at rest or partial pressure of oxygen $<60 \mathrm{mmHg}$ at arterial blood gas analysis*; respiratory rate $>30$ breaths / $\mathrm{min}$; new onset of dyspnea at rest or during speaking; reduction of oxygen saturation on room air below $90 \%$ during walking test; high value of prognostic scores; presence of anuria, confusion, hypotension, cyanosis, and/or other medical conditions requiring hospitalization per se-best practice recommendation (based on expert opinion only)

*This does not strictly apply to patients with chronic obstructive pulmonary disease or other chronic respiratory disease, in whom similar values may be well tolerated, but who nonetheless need a careful personalized evaluation for hospitalization considering the presence of a baseline respiratory disease besides COVID-19

Question 2 Which drugs should be administered to outpatients with COVID-19?

Based on available results from RCTs, we do not recommend the administration of hydroxychloroquine in outpatients with COVID-19-strong recommendation, moderate certainty of evidence

We do not recommend the use of corticosteroids in outpatients with COVID-19, unless needed for other medical reasons-best practice recommendation (based on expert opinion only)

In the absence of proven bacterial infections, the administration of antibiotics in outpatients with COVID19 should be considered only as empirical treatment of highly suspected bacterial co-infection or superinfections-weak recommendation, very low certainty of evidence (for azithromycin); best practice recommendation for other antibiotics (based on expert opinion only)

At the present time, antivirals should not be administered in outpatients with COVID-19 outside RCTsbest practice recommendation (based on expert opinion only)

The use of neutralizing monoclonal antibodies may be considered in outpatients with COVID-19 with $\mathrm{mild} /$ moderate diseases at risk of progression and within at most 10 days after symptoms onset-weak recommendation, low certainty of evidence

Of note, there was some agreement across the panel regarding the possibility to consider colchicine for the treatment of selected subgroups of outpatients with COVID-19, provided the favorable results in patients with positive COVID-19 molecular test in the COLCORONA RCT are replicated in other studies [66] 
Table 1 continued

\section{Question Recommendations}

Question 3 Should anticoagulant agents be administered to inpatients with COVID-19?

Unless contraindicated, we recommend prophylactic anticoagulation in hospitalized patients with COVID19-strong recommendation, low certainty of evidence

Hospitalized patients with COVID-19 who were already under chronic anticoagulant therapy for welldefined indications, unless contraindicated, should continue anticoagulant treatment-best practice recommendation (based on expert opinion only)

Therapeutic anticoagulation may be considered in patients possibly at higher risk of thrombotic events (serum d-dimer levels $>2.0 \mu \mathrm{g} / \mathrm{mL}$ ) or with high suspicion for thrombotic complications-best practice recommendation (based on expert opinion only)

These recommendations are intended for inpatients with COVID-19 outside ICU

Question 4 Should systemic steroids be administered to inpatients with COVID-19?

Unless contraindicated, we recommend the use of dexamethasone at the dosage of $6 \mathrm{mg} /$ day for 10 days in inpatients with COVID-19 requiring oxygen supplementation*-weak recommendation, low certainty of evidence

Methylprednisolone at the dosage of $0.5 \mathrm{mg} / \mathrm{kg}$ twice daily for at least 5 days could be considered in inpatients with COVID-19 requiring oxygen supplementation and aged 60 years or older-weak recommendation, very low certainty of evidence

These recommendations are intended for inpatients with COVID-19 outside ICU

${ }^{*}$ Equivalent dosages of other steroids may be considered if dexamethasone is not available (although this should be considered as best practice recommendation, taking also into account the indirectness of evidence for steroids other than dexamethasone)

Question 5 Should antiviral agents be administered to inpatients with COVID-19?

Lopinavir/ritonavir should not be administered to hospitalized patients with COVID-19-strong recommendation, moderate certainty of evidence

Pending further results from large RCTs, administration of a 5-day course of remdesivir should be considered in hospitalized patients with COVID-19 pneumonia requiring oxygen supplementation -weak recommendation, very low certainty of evidence

Hydroxychloroquine should not be administered to hospitalized patients with COVID-19—strong recommendation, moderate certainty of evidence

Other antiviral agents should not be administered for treating COVID-19 in hospitalized patients, unless they are administered within RCTs-best practice recommendation (based on expert opinion only)

These recommendations are intended for inpatients with COVID-19 outside ICU 
Table 1 continued

\section{Question Recommendations}

Question 6 Should antibiotics be administered to inpatients with COVID-19?

We recommend against the routine use of antibiotics in hospitalized patients with COVID-19 without proven bacterial infection-strong recommendation, moderate certainty of evidence (for azithromycin); weak recommendation, very low certainty of evidence (for antibiotics in general)

We recommend collection of respiratory specimens for culture or molecular detection of respiratory pathogens, blood cultures, and urinary antigens for Streptococcus pneumoniae and Legionella spp. in hospitalized patients with COVID-19 and suspected bacterial pneumonia-best practice recommendation (based on expert opinion only)

Empirical antibiotic treatment of suspected bacterial pneumonia alongside proper diagnostic procedures, should be considered in patients with COVID-19 with evidence of consolidative radiological lesions-best practice recommendation (based on expert opinion only)

In the case of empirical antibiotic treatment, selection of agents to be administered should follow standard practice for the treatment of bacterial pneumonia-best practice recommendation (based on expert opinion only)

These recommendations are intended for inpatients with COVID-19 outside ICU

Question 7 Should neutralizing monoclonal antibodies and non-steroid immunomodulators be administered to inpatients with COVID-19?

Pending further results from RCTs, we recommend against the administration of neutralizing monoclonal antibodies in hospitalized patients with COVID-19-strong recommendation, moderate certainty of evidence

We recommend considering tocilizumab administration in hospitalized patients with COVID-19 not responding to steroid treatment, with oxygen saturation $<92 \%$ on room air (including those already on supplementary oxygen), and with increased inflammatory markers* in the absence of a proven or suspected bacterial or fungal infection ${ }^{* *}$-weak recommendation, very low certainty of evidence

Pending further results from RCTs, baricitinib may be considered in addition to remdesivir in patients requiring high-flow oxygen or non-invasive mechanical ventilation who are not under steroid treatment (e.g., in the presence of contraindications to steroid use)-weak recommendation, low certainty of evidence

Pending further results from large RCTs, we recommend against administration of other non-steroid immunomodulatory agents outside RCTs-weak recommendation, very low certainty of evidence (for anakinra); best practice recommendation for other agents (based on expert opinion only)

These recommendations are intended for inpatients with COVID-19 outside ICU

${ }^{*}$ In the RECOVERY trial, serum C-reactive protein $\geq 75 \mathrm{mg} / \mathrm{L}$

${ }^{* *}$ Clinicians should be aware of the following: (i) the $75 \mathrm{mg} / \mathrm{L}$ cutoff is based on results of the RECOVERY RCT; (ii) other markers of inflammation may be considered on a case-by-case basis (best practice recommendation); (iii) another best practice recommendation is to avoid tocilizumab administration in patients with severe immunosuppression or in those with other contraindications to tocilizumab administration (low platelet count; risk of gastrointestinal perforation; increase of transaminases $>5$ times the upper limit of normal) 
Table 1 continued

\section{Question Recommendations}

Question 8 Should convalescent plasma be administered to inpatients with COVID-19?

Pending further results from RCTs, currently we do not support the administration of convalescent plasma in hospitalized patients with COVID-19 outside RCTs-weak recommendation, low certainty of evidence

Pending further results from RCTs, currently we do not support the administration of anti-COVID-19 hyperimmune immunoglobulin preparations in hospitalized patients with COVID-19 outside RCTs-best practice recommendation (based on expert opinion only)

These recommendations are intended for inpatients with COVID-19 outside ICU

Question 9 Should CPAP/NIV be employed for treating inpatients with COVID-19 with acute hypoxemic respiratory failure?

Unless contraindicated, non-invasive ventilatory support by means of NIV or CPAP is feasible and safe in patients with acute respiratory failure secondary to COVID-19, and should be considered for patients in whom standard oxygen supplementation is not or no longer sufficient and who do not require immediate intubation-best practice recommendation (based on expert opinion only)

CPAP delivery systems allowing for PEEP titration should be preferred, and PEEP should not exceed $10 \mathrm{cmH}_{2} \mathrm{O}$-best practice recommendation (based on expert opinion only)

These recommendations are intended for inpatients with COVID-19 outside ICU

Question 10 When can an improved patient with COVID-19 be discharged from an acute care hospital?

Clinically stable patients with COVID-19 who no longer require isolation (or who can be isolated outside the hospital) should be discharged from acute care hospitals when oxygen supplementation is no longer required or with a maximum requirement of low-flow oxygen at $2 \mathrm{~L} / \mathrm{min}$ through nasal cannula (with the exception of patients already under oxygen supplementation at home at baseline or patients requiring initiation of long-term oxygen therapy after discharge), in line with common practice with other types of non-contagious lower respiratory tract infections, and provided there are no complications or other reasons that require continuation of hospitalization-best practice recommendation (based on expert opinion only)

For patients with COVID-19 still requiring isolation but who could be discharged from a clinical standpoint, isolation outside the hospital (at home, in community facilities, or in long-term facilities, according to the specific need for non-acute care of any given patient) should be supported and made feasible for as many patients as possible-best practice recommendation (based on expert opinion only)

COVID-19 coronavirus disease 2019, CPAP continuous positive airway pressure, NIV non-invasive ventilation, PEEP positive end-expiratory pressure, $R C T s$ randomized controlled trials

the population is composed only of hospitalized patients; therefore, it may remain unclear as to whether patients with a favorable outcome would have experienced the same outcome without hospital care. In other words, there is no assessment of the efficacy of hospitalization (considered as an intervention) with respect to clinical outcomes. Together with the lack of evidence from RCTs, this is a major limitation when assessing the available evidence for replying to the present question.

Notwithstanding the aforementioned limitations, the available literature provides some information about the prognostic performance in patients with COVID-19 of known severity scores for community-acquired pneumonia, 
such as the pneumonia severity index (PSI) and the CURB-65 score, that may provide some rationale on their possible use for deciding in favor or against hospitalization (although with the relevant bias of being assessed in inpatients and not in outpatients) [29-32]. In a recent single-center prospective study including 249 patients with COVID-19 in Spain [32], PSI assessed at hospital admission showed overall good accuracy for predicting case fatality (area under the receiver operating characteristic curve [AUC ROC] of 0.87 , with $95 \%$ confidence intervals $[\mathrm{CI}]$ 0.81-0.94). However, up to $37.7 \%$ patients with low PSI scores (I-III) showed progression to severe disease, likely making this tool unreliable for decisions on hospitalization. Similar results were observed for the CURB-65 score, with $26.6 \%$ of patients with a low CURB65 score (0-2) progressing to severe disease. In another study of 208 patients with COVID-19, all with a low CURB-65 score of 0-2, 40 (19.2\%) patients progressed to severe diseases [31].

Other scoring systems have been proposed for stratifying patients with COVID-19 at high or low risk for disease progression and/or poor outcome at the time of hospital admission [31-34]. The MuLBSTA score is a fatality prediction score for viral pneumonia that includes six variables, namely multilobal infiltration, lymphopenia, bacterial co-infection, smoking history, age, and hypertension [32, 34, 35]. The MuLBSTA score has also been proposed to avoid hospitalization in patients with COVID-19, since none of the patients with less than five points showed disease progression. However, the cohort was composed of hospitalized patients and the sample size was small $(n=72)$ [34]. Another prognostic score, named CALL and developed in a cohort of 208 hospitalized patients with COVID-19, is based on comorbidities, age, blood lymphocyte count, and serum lactate dehydrogenase (LDH) value [31]. Overall, patients with a low CALL score of 4-6 points had less than $10 \%$ probability of progression to severe disease [31], but a formal assessment of this score in terms of internal and external validity is needed [36]. The National Early Warning Score-2 (NEWS-2) based on six physiological parameters was also evaluated for its prognostic performance in hospitalized patients with COVID-19, but any possible impact on hospitalization decisions remains to be assessed [33, 37, 38]. Besides the aforementioned, also many other prognostic scores/models have been proposed that, for the purpose of the present question, suffer from the same limitation, i.e., it is not possible to directly extrapolate their performance to hospitalization decisions [39-53].

Finally, it should be noted that the characteristics of patients admitted to hospitals are highly variable across different healthcare systems [30] and among the same healthcare system the threshold for hospitalization may vary according to the burden of disease and resource availability. In addition, clinical severity is not the only variable guiding hospital admission. Indeed, some patients may have social contraindications to outpatient management, such as inability to maintain oral intake, fiduciary isolation, or impaired functional status. Finally, there is still no evidence that implementation of severity tools into clinical practice results in better prognosis, lower costs, and lower mortality in patients with COVID-19. None of the available tools has been currently fully validated and is ready for widespread implementation in clinical practice [30-35].

\section{Conclusive Remarks}

The currently available evidence does not allow us to provide recommendations based on the GRADE system regarding the criteria of hospitalization for patients with COVID-19, owing to the lack of studies adequately assessing the impact of hospitalization as an intervention (overall and in specific subgroups). While further research efforts certainly remain necessary in this area, in the opinion of the panel it is nonetheless reasonable to set temporary hospitalization criteria (taking into account the unfavorable prognostic effect of several parameters), which are provided below as a best practice recommendation, and that follow the criteria recently released by the Italian Ministry of Health, based on expert consensus [54]. As an expression of expert consensus, we acknowledge that these criteria may be subject to modification should further evidence become available in the near future. 


\section{Recommendations}

- Pending further evidence, it might be prudent not to base the decision to hospitalize or not patients with COVID-19 only on prognostic scores-weak recommendation, very low certainty of evidence

- Hospitalization should be considered in patients with at least one of the following: low oxygen saturation on room air $\leq 92 \%$ at rest or partial pressure of oxygen $<60 \mathrm{mmHg}$ at arterial blood gas analysis*; respiratory rate $>30$ breaths /min; new onset of dyspnea at rest or during speaking; reduction of oxygen saturation on room air below $90 \%$ during walking test; high value of prognostic scores; presence of anuria, confusion, hypotension, cyanosis, and/or other medical conditions requiring hospitalization per se-best practice recommendation (based on expert opinion only)

*This does not strictly apply to patients with chronic obstructive pulmonary disease or other chronic respiratory disease, in whom similar values may be well tolerated, but who nonetheless need a careful personalized evaluation for hospitalization considering the presence of a baseline respiratory disease besides COVID-19.

\section{Future Research Directions}

- To assess the impact of hospitalization as intervention in specific and homogeneous subgroups of patients with mild COVID-19 in which the potential benefit of hospitalization remains unclear

- To develop and to validate novel hospitalization scores based on the results of studies assessing the impact of hospitalization as intervention

\section{Question 2: Which Drugs Should be Administered to Outpatients with COVID- 19 ?}

\section{Evidence Summary}

Most literature on the pharmacological treatment of patients with COVID-19 pertains to hospitalized patients. This can be attributed to many reasons, including (1) frequent unavailability of several data (laboratory results, clinical charts) for outpatients with mild disease; (2) a more clear definition of subgroups in hospitalized patients (i.e., of different phenotypes based on laboratory/radiology data); (3) an expected higher frequency of loss at follow-up in outpatients; (4) the low frequency of critical outcomes such as death in outpatients with COVID-19 (in whom mild forms are expected to predominate). For this last reason, in this review, although mortality remained an important outcome, we focused also on other relevant outcome measures (hospitalization, worsening of clinical conditions). It is also important to note that the population of interest for this question was patients with mild (or in some cases moderate) disease not requiring hospitalization. In this regard, we acknowledge that some patients requiring hospitalization may be not hospitalized (or not hospitalized promptly) in the case of exponential increases in the number of cases and overcrowded hospitals. However, this should not be the rule, and efforts should be directed towards preventing further excessive increases in the number of severe cases through efficient vaccination campaigns.

Despite the smaller amount of literature on outpatients with COVID-19 as compared to inpatients, for the present question the guidelines panel decided to rely on high-level evidence from RCTs, after having verified the absence of homogenous directions of effect combined with large effect sizes in large observational studies. There was indeed a large agreement across panel members that therapeutic decisions in outpatients (in a large portion of whom COVID-19 is expected to be a selflimiting disease) should be guided by clear evidence of a possible benefit, in line with the principle of "first, do not harm". More details regarding the RCTs discussed in this section are available in the Supplementary Material.

It should also be noted that our literature selection was initially focused on RCTs published in peer-review journals. However, based on the possibility of impacting clinical practice, we also discussed the results of a large RCT on the efficacy and safety of colchicine in 
outpatients with COVID-19, which was available only on a pre-print server at the time of revision but that has been subsequently published in its final peer-reviewed form (see below).

While preliminary observational, retrospective experiences including outpatients with COVID-19 suggested a possible beneficial effect of hydroxychloroquine in terms of mortality or reduced rates of hospital admission [55, 56], these hypothesis-generating findings were not confirmed in subsequent randomized studies. The efficacy of hydroxychloroquine in outpatients with COVID-19 was assessed in a multicenter, open-label RCT trial conducted in Spain [57]. Enrolled patients were non-hospitalized adults with COVID-19 and less than 5 days of symptoms. No differences between the two arms were observed in the primary efficacy endpoint (reduction of viral load in nasopharyngeal swabs). As regards secondary endpoints, hydroxychloroquine did not reduce the risk of hospitalization, nor did it shorten the median time to complete resolution of symptoms. In a double-blind RCT, conducted in symptomatic, non-hospitalized adults with COVID-19, no differences were observed between hydroxychloroquine and placebo with respect to the primary endpoint, an ordinal 10-point severity scale [58]. Results of further RCTs published in 2021 are in line with the absence of a favorable effect of hydroxychloroquine in outpatients with mild COVID-19 [59, 60].

We found no large RCTs about the use of oral or intravenous steroids in outpatients addressing the outcomes of interests. Nonetheless, we think that some indirect evidence can be extrapolated from results of RCTs conducted in hospitalized patients with mild forms of COVID-19. More specifically, in a controlled, open-label trial, hospitalized patients with COVID-19 were randomly assigned to receive dexamethasone orally or intravenously at the dosage of $6 \mathrm{mg}$ once daily for up to 10 days or to receive usual care alone [61]. The primary outcome measure was death within 28 days after randomization. In this study, mortality was lower in the dexamethasone group than in the usual care group among patients receiving oxygen (see question 4), but not among patients with milder forms not receiving oxygen. In our opinion, although indirectly, this finding, i.e., a lack of effect in patients with mild forms not requiring oxygen supplementation, can be extrapolated to outpatients, who usually share these features. Regarding inhaled steroids, in a recent open-label, phase 2 RCT including 146 participants, budesonide was compared to standard care alone in adults within 7 days of mild COVID-19 symptoms onset [62]. The primary endpoint was COVID-19-related urgent care visit, with a lower proportion of urgent visits being observed in the budesonide arm (1\% vs. $14 \%$ ). Keeping in mind the limitations connected to the limited sample size (which in part remain although related to early termination), this favorable result should prompt further dedicated investigation and validation [63].

Azithromycin was one of the most common antibiotics administered to patients with COVID-19 in the first months of the pandemic, based on some preliminary exploratory studies reporting a possible beneficial effect. According to the currently available evidence, the use of azithromycin does not improve clinical status in patients with mild COVID-19. At the end of our first literature review, as for steroids, the evidence supporting this statement could only be indirectly extrapolated to outpatients from RCTs conducted in inpatients, showing no beneficial effect [64]. However, the results of an open-label RCT conducted in outpatients with suspected COVID-19 aged 65 years or older (or at least 50 years with at least one comorbidity) and comparing azithromycin in addition to usual care vs. usual care alone recently became available [65]. Azithromycin was not associated with better time to first reported recovery compared with usual care alone (hazard ratio [HR] 1.08, with 95\% Bayesian credibility interval from 0.95 to 1.23). Sixteen out of 500 outpatients (3\%) in the azithromycin arm and 28 out of 823 outpatients (3\%) in the usual care alone arm were hospitalized (absolute benefit in percentage $0.3 \%$, with $95 \%$ Bayesian credible intervals from -1.7 to 2.2 ). Overall, it should be noted that only $1148 / 1388$ subjects (83\%) had a SARS-CoV-2 molecular result available, and only 434 subjects (31\% of the entire population) had a positive result. Non-superiority 
of azithromycin was also observed in a subgroup analysis in confirmed COVID-19 cases, which is consistent with the primary study results, although a larger imprecision of estimates should be taken into account.

The efficacy and safety of colchicine for the treatment of outpatients aged 40 years or older, with suspected/proven COVID-19, and with a least one risk factor for disease progression (see the Supplementary Material for details) was assessed in a double-blind RCT [66]. Although not reaching superiority in the entire cohort (suspected or proven infections), in the subgroup of outpatients with proven COVID-19 colchicine (administered for 1 month) was associated with a reduction in the risk of death or hospitalization, which was the primary composite efficacy endpoint (occurring in 4.6\% and $6.0 \%$ of patients in colchicine and placebo arms, respectively). While no significant difference was observed in the overall study population and the small number of events in the cohort advocate for caution and against claims of large therapeutic effects, it is opinion of the panel that colchicine may be considered for the treatment high-risk outpatients, provided the results of this trial are confirmed in other RCTs of outpatients with proven COVID-19. Of note, in a previous small RCT, colchicine was associated with improved time to clinical deterioration in hospitalized patients not requiring oxygen supplementation [67]. However, the small sample of the trial does not allow either generalization to hospitalized patients or extrapolation to outpatients (based on the fact that colchicine was administered to hospitalized patients with mild disease).

Recently, the results of RCTs assessing the efficacy and safety of neutralizing monoclonal antibodies in outpatients with COVID-19 were published [68-70]. The main target of SARSCoV-2 neutralizing monoclonal antibodies is the surface spike glycoprotein that mediates viral entry into host cells. The neutralizing monoclonal antibodies in late phases of clinical development and approved by the US Food and Drug Administration (FDA) for emergency use are (1) the combination of bamlanivimab (also known as LY-CoV555) and etesevimab (also known as LY-CoV016), which consists of antibodies directed against different portions SARS-CoV-2 spike protein and its receptor binding domain (RBD); (2) REGN-COV2, which is the combination of the two monoclonal antibodies casirivimab (REGN10933) and imdevimab (REGN10987), which are designed to bind different regions of the SARS-CoV-2 spike protein RBD. Overall, the results of published RCTs (detailed in the Supplementary Material) show an accelerating effect of both combinations on the natural decrease of SARSCoV-2 viral load, as well as a possible favorable effect in reducing progression to hospitalization [68-70]. Of note, the effect of bamlanivimab/ etesevimab in reducing hospitalization was maximized in patients aged 65 years of age or older or with a body mass index of 35 or greater (although the latter results stem from post hoc subgroup analyses) [69]. The Italian Medicine Agency (AIFA) recently granted a conditional approval for the use of neutralizing monoclonal antibodies in outpatients at risk within at most 10 days after symptoms onset, considering the current absence of drugs of proven efficacy for this specific setting (outpatients with COVID-19 at risk of progression) [71]. The opinion of the guidelines panel is in line with such a conditional use provided that risk factors for progression are present. Nonetheless, it should be stressed (as also recognized by AIFA) that uncertainties remain about the true magnitude of the effect, and that the favorable efficacy data are still preliminary. For this reason, the current certainty of evidence was ultimately considered low.

We did not retrieve large, peer-reviewed RCTs on the possible favorable/unfavorable effects of antivirals (except for a large RCT showing no effect of lopinavir/ritonavir compared with placebo with respect to the risk of hospitalization [60]), convalescent plasma (one study was only partially conducted in outpatients, see question 8), and prophylactic antithrombotic agents in outpatients with COVID-19 (except for preliminary favorable results for sulodexide, to be further confirmed) [72]. Finally, the possible role of ivermectin, an antiparasitic drug showing some in vitro activity against SARS-CoV-2 [73], remains unclear, with conflicting evidence and still not 
convincing support for its use stemming from currently available RCTs [74-78].

\section{Conclusive Remarks}

A strikingly small number of published RCTs that assess the pharmacological treatment of COVID-19 in outpatients are presently available. This prompted the panel not to currently support many pharmacological treatments for COVID-19 (in line with the principle of "first, do not harm" in patients with mild disease presentation), with the exception of neutralizing monoclonal antibodies in specific situations, the possible exception of colchicine (especially after a future replication of the favorable results in patients with positive COVID-19 molecular test in the COLCORONA RCT [66]), and the exception of symptomatic use of antipyretic and analgesic agents. The panel nonetheless remains very open to possible future modifications of the recommendations, provided high-quality results from large RCTs show a clear beneficial effect either in the entire population or in specific subgroups of outpatients with COVID-19.

\section{Recommendations*}

- Based on available results from RCTs, we do not recommend the administration of hydroxychloroquine in outpatients with COVID-19-strong recommendation, moderate certainty of evidence

- We do not recommend the use of corticosteroids in outpatients with COVID-19, unless needed for other medical reasonsbest practice recommendation (based on expert opinion only)

- In the absence of proven bacterial infections, the administration of antibiotics in outpatients with COVID-19 should be considered only as empirical treatment of highly suspected bacterial co-infection or superinfections-weak recommendation, very low certainty of evidence (for azithromycin); best practice recommendation for other antibiotics (based on expert opinion only)

- At the present time, antivirals should not be administered in outpatients with COVID-19 outside RCTs-best practice recommendation (based on expert opinion only)

- The use of neutralizing monoclonal antibodies may be considered in outpatients with COVID-19 with mild/moderate diseases at risk of progression and within at most 10 days after symptoms onset-weak recommendation, low certainty of evidence

* Of note, there was some agreement across the panel regarding the possibility to consider colchicine for the treatment of selected subgroups of outpatients with COVID-19, provided the favorable results in patients with positive COVID-19 molecular test in the COLCORONA RCT are replicated in other studies [66].

\section{Future Research Directions}

- To increase the number of large RCTs addressing the possible favorable impact of pharmacological treatments in outpatients with COVID-19 (overall and in different subgroups according to the risk of disease progression)

- To provide results from large RCTs addressing the possible use of antiviral and prophylactic antithrombotic agents in outpatients with COVID-19 (overall and according to the risk of disease progression)

\section{Question 3: Should Anticoagulant Agents be Administered to Inpatients with COVID-19?}

\section{Evidence Summary}

Thrombotic complications may contribute to the morbidity and mortality of hospitalized patients with COVID-19, through various nonmutually exclusive mechanisms such as venous thromboembolism, arterial thrombosis, and thrombotic microangiopathy [79]. Consequently, prophylaxis and treatment of thrombotic complications with anticoagulant agents are largely employed in the management of hospitalized patients with COVID-19.

The literature review for the present section was performed taking into account some key concepts. First, the population of interest was represented by hospitalized patients with 
COVID-19 outside ICU. Second, this population could be conceptually divided into two subpopulations: (1) hospitalized patients with COVID-19 who have already developed thrombotic complications, and thus already require anticoagulant agents at therapeutic dosage, and in some cases fibrinolysis [80]; (2) hospitalized patients with COVID-19 with no evidence of thrombotic complications, in whom anticoagulant agents might be useful to prevent the development of thrombotic complications. We decided to focus on this latter subpopulation, in an attempt to answer the following clinically relevant questions: (1) Do these patients require administration of anticoagulant agents? (2) If they require anticoagulant agents, should we administer them at prophylactic or at therapeutic dosages? (3) If they require anticoagulant agents, should a specific anticoagulant agent/class be preferred to others?

To answer the first question (do these patients require administration of anticoagulant agents?), we searched for studies in which the administration of anticoagulant agents was compared to no administration of any of these agents. To answer the second question (if they require anticoagulant agents, should we administer them at prophylactic or therapeutic dosages?), we searched for studies in which the intervention was receipt of anticoagulant agents at prophylactic dosage and the comparator was receipt of anticoagulant agents at therapeutic dosage, or vice versa. To answer the third question (if they require anticoagulant agents, should a specific anticoagulant agent be preferred over others?), we searched for studies in which the intervention was receipt of a given anticoagulant agent and the comparator was receipt of another/other anticoagulant agent/s. For all questions, we defined the clinically relevant efficacy endpoints of interest to be (1) mortality; (2) need for ICU admission; (3) development of thrombotic complications. An additional safety endpoint of clinical interest was development of hemorrhagic complications.

The ideal situation would have been to base our answers to these three questions on highquality evidence from randomized studies. However, no peer-reviewed RCTs conducted outside ICU were initially retrieved from the literature search, although it is worth noting that the results of an interim analysis combining three different RCTs (ATTACC, ACTIV-4, REMAP-CAP) has recently become available as two pre-print manuscripts, showing an advantage, for anticoagulant prophylaxis, of anticoagulants at therapeutic dosages vs. standard prophylactic dosages in patients with moderate COVID-19, independent of d-dimer values, and, conversely, an apparent lack of benefit in patients with severe COVID-19 (ICU level of care) receiving therapeutic dosages of anticoagulants [81-83]. Although still to be peer-reviewed (and thus not considered as highcertainty evidence) these results were taken into account for drafting recommendations. Of note, a published RCT was not considered for the present question as conducted exclusively in ICU patients [84]. Finally, the results of the ACTION RCT, which became available just before release of the present guidelines, showed no advantage of therapeutic vs. prophylactic dosages of anticoagulants (enoxaparin followed by oral rivaroxaban, or, mostly, rivaroxaban alone) for improving clinical outcomes of hospitalized patients with COVID-19 and elevated d-dimer concentrations [85]. This study will deserve global discussion in future versions of the present document when full peer-reviewed results of the other RCTs introduced above will be available, also with the aim of comparing different possible anticoagulant regimens.

The majority of studies retrieved in our literature search were observational. Most of them were retrospective $(33 / 35,94 \%)$ [86-118]. Only two studies were prospective $(6 \%)[119,120]$. A brief summary of the retrieved evidence is presented in the following paragraphs and in Table S1 in the Supplementary Material, whereas an extended summary of the characteristics and results of all included studies is available in the Supplementary Material.

Before proceeding with the summary and discussion of evidence, some difficulties we found in organizing the available evidence should be acknowledged: (1) it was not always possible to limit the study populations to nonICU inpatients; (2) in many studies, it was unclear whether admission to ICU was a 
baseline condition (before initiation of anticoagulant agents) or an outcome (i.e., occurring after initiation of anticoagulant agents); (3) it was not always possible to limit the population to patients without already existing thrombotic events; (4) in light of the previous point, in many studies it was unclear whether thrombotic complications occurred in patients already receiving therapeutic anticoagulants or they were instead the reason for the administration of therapeutic anticoagulants; (5) composite endpoints were ultimately not included in our review because of their heterogeneity across studies; (6) the impact of pre-existing outpatient use of anticoagulants for other conditions was not assessed because of the frequent lack of adjustment for in-hospital initiation/changes of anticoagulant agents, and the related recommendation was thus based on panel opinion only; (7) finally, considering the frequent presence of data on in-hospital use of anticoagulants in the literature on inpatients with COVID-19, some studies meeting inclusion criteria may have not been caught by our search, especially if not focused on the use of anticoagulant agents.

Comparison of Anticoagulant Agents vs. No Anticoagulant Agents Overall, 22 observational studies (20 retrospective and 2 prospective) compared administration of anticoagulant agents vs. no administration of anticoagulant agents with respect to different primary endpoints [86-90, 92, 94-96, 100, $102,104-111,113,117,119]$. The most frequent primary endpoint (17/22 studies, $77 \%)$ was in-hospital mortality. Despite differences in study designs and populations (see details for each study in Supplementary Material), some general information and trends can be extrapolated: (1) most patients received anticoagulant agents at prophylactic dosages; (2) the most frequently employed anticoagulant agents were low molecular weight heparins (LMWH); (3) a favorable effect in terms of reduced mortality in adjusted analyses was observed in patients receiving anticoagulant agents vs. no anticoagulant agents in most studies assessing mortality as the primary/major endpoint (see Fig. S1 in the Supplementary Material). Subgroup analyses on the impact of anticoagulant agents according to different serum d-dimer levels were available in a few studies [89, 104, 106, 111], overall showing a possible lack of or a less marked protective effect of anticoagulant agents on mortality in patients with serum d-dimer lower than $1-3 \mu \mathrm{g} / \mathrm{mL}$, although a clear cutoff for serum d-dimer remains to be established.

Regarding admission to ICU, we were eventually unable to retrieve reliable information regarding the possible protective effect of anticoagulant agents. Indeed, although the distribution of ICU admissions between patients receiving anticoagulant agents and patients not receiving anticoagulant agents was available in as many studies, in most cases it was unclear whether ICU admission was a baseline condition or a true endpoint. Furthermore, ICU admission was among the primary endpoints only rarely, and in these studies the comparison between patients receiving and not receiving anticoagulant agents was not adjusted for potential confounding factors (see Table S1 in the Supplementary Material).

Four out of 22 studies (18\%) investigated development of thrombotic complications as a major endpoint $[94,96,105,108]$. Only in two of them was a multivariable analysis presented, showing a protective effect of anticoagulant agents (Table S1 in the Supplementary Material). Details regarding effect sizes with respect to the primary endpoints in the different studies are available in Table S1 in the Supplementary Material.

Comparisons of Anticoagulant Agents at Prophylactic Dosage vs. Anticoagulant Agents at Therapeutic Dosage and Comparisons Between Different Anticoagulant Agents Overall, only 15/35 studies (43\%) [91, 93, 97-99, 101, 103, 106, 109, 112, 114-118], all retrospective, could be included in this section, of which 13 compared prophylactic dosages vs. subtherapeutic/therapeutic dosages and only two compared prophylactic enoxaparin vs. prophylactic fondaparinux, showing no striking differences in the performed unadjusted comparisons $[101,118]$. With regard to the comparison of prophylactic vs. therapeutic dosages, mortality was the endpoint (or one of the endpoints) of 
major interest in 9/13 studies (69\%), in most cases with no relevant differences between prophylactic dosages and therapeutic dosages in adjusted comparisons (when available). In two studies, the primary endpoint was development of hemorrhagic complications, with higher dosages being possibly associated with increased risk of bleeding in comparison with standard prophylactic dosages. Details regarding effect sizes with respect to the primary endpoints reported in the different studies are available in Table $S 1$ in the Supplementary Material.

\section{Conclusive Remarks}

Overall, despite the low certainty of evidence inherent in the observational nature of most retrieved studies, a general trend toward reduced mortality in patients receiving anticoagulant agents (mainly at prophylactic dosages) was appreciable in studies with mortality as a primary endpoint. This finding, in our opinion, may support a strong recommendation towards the administration of prophylactic anticoagulants despite low certainty of evidence. This recommendation is supported by the following consequent considerations: (1) in the large majority of studies the reduction in mortality associated with the use of anticoagulant agents was more marked or present only in multivariable analyses, after adjustment for potential confounding factors; (2) this finding indicates that the use of anticoagulant was more likely in patients at worse prognosis; (3) therefore, it may be speculated that any residual confounding unaccounted for in multivariable analyses would introduce a bias against the efficacy of anticoagulant agents.

Considering the available evidence and the need for evaluating full peer-reviewed data from recently completed RCTs (see above), no recommendations based on GRADE criteria were made in the present version of the guidelines about the possible prophylactic use of higher dosages of anticoagulant agents in patients with COVID-19 without thrombotic complications, either in general or according to d-dimer values (only best practice recommendations are provided, to be reassessed the future). The same applies to the choice among different anticoagulant agents.

\section{Recommendations*}

- Unless contraindicated, we recommend prophylactic anticoagulation in hospitalized patients with COVID-19-strong recommendation, low certainty of evidence

- Hospitalized patients with COVID-19 who were already under chronic anticoagulant therapy for well-defined indications, unless contraindicated, should continue anticoagulant treatment-best practice recommendation (based on expert opinion only)

- Therapeutic anticoagulation may be considered in patients possibly at higher risk of thrombotic events (serum d-dimer levels $>2.0 \mu \mathrm{g} / \mathrm{mL}$ ) or with high suspicion for thrombotic complications-best practice recommendation (based on expert opinion only)

* These recommendations are intended for inpatients with COVID-19 outside ICU.

\section{Future Research Directions}

- To further assess the differential efficacy and safety of different anticoagulant agents and different dosages of the same anticoagulant agents, preferably in RCTs

- To further evaluate the role of serum d-dimer levels and/or other laboratory markers in guiding decision about both administration and dosage of anticoagulant agents

\section{Question 4: Should Systemic Steroids be} Administered to Inpatients with COVID19 ?

\section{Evidence Summary}

The rationale for administering systemic steroids in patients with COVID-19 is their antiinflammatory effect (that on the one hand could be beneficial by counteracting excessive inflammation, but on the other hand could be detrimental by hampering the natural host response to the virus). At the beginning of the COVID-19 pandemic, the use of systemic steroids for the treatment of severe coronavirus disease was a highly controversial topic owing 
to an unclear balance between possible benefits and arms in previous small experiences in patients with severe pulmonary infections due to SARS-CoV-1 or MERS-CoV [121, 122]. After some exploratory, observational experiences in patients with COVID-19 (some examples are [123-128]), the results of the RECOVERY RCT showed a favorable effect of dexamethasone (6 mg daily for 10 days) in terms of survival in two subgroups of hospitalized patients with COVID-19 (i.e., patients requiring invasive mechanical ventilation and those not requiring invasive mechanical ventilation but needing oxygen supplementation, whereas no effect was observed in patients not requiring supplementary oxygen) [61]. Besides the RECOVERY RCT, our literature search initially led to the inclusion of four other RCTs addressing (as primary population or, more often, in subgroup analyses) the efficacy of systemic steroids in inpatients with COVID-19 not subjected to invasive mechanical ventilation. The choice of patients not subjected to invasive mechanical ventilation instead of ICU patients as the population of interest for the present question was based on the frequent stratification in subgroups according to the type of ventilatory support instead of ward of stay. Thus, it was taken into account by the panel that the present review may have in part implied an indirect extrapolation of results in non-invasively ventilated patients with severe COVID-19 in ICU to non-invasively ventilated patients outside ICU. Two RCTs assessed the efficacy of systemic methylprednisolone administration in patients with COVID-19 not undergoing invasive mechanical ventilation, showing conflicting results (one reported a favorable effect on mortality and the other one did not) $[129,130]$. The reasons for this difference remain partly unclear and include, beside the small sample of at least one of the two studies, differences in study design, such as the different inclusion criteria, the different doses of the drug, and the timing of administration. However, these factors seem unable to completely explain the fact that, in the larger of the two studies, no beneficial effect of methylprednisolone was observed (in post hoc analyses) either among intubated or non-intubated patients. Conversely, in the same trial there was an apparent age-dependent efficacy, i.e., a favorable effect of methylprednisolone on survival in patients aged 60 years or older. A third, small, open-label RCT including 64 patients was recently published and retrieved during the latest update of our search; that RCT compared methylprednisolone vs. standard of care alone, showing a possible advantage of methylprednisolone with respect to the primary composite endpoint of death, admission to the ICU, or requirement for non-invasive ventilation [131]. In another small RCT (86 patients), need for ventilation was registered in $18 \%$ and $38 \%$ of patients in methylprednisolone and dexamethasone arms, respectively [132]. Two other RCTs were conducted in ICU that included also patients not subjected to invasive mechanical ventilation at baseline [133, 134]. Although they were conducted in the ICU and not in the population of interest for the present review, it is of interest that also for these two trials results were somewhat conflicting. Indeed, although both RCTs were interrupted early, enrollment was discontinued for futility in one case and after the release of the positive results of the RECOVERY trial in the other one (in this latter RCT, although a significant effect was not observed in the entire population, a possible favorable effect of a fixed-dose 7-day course of hydrocortisone was detected in pre-planned subgroup analyses in patients not receiving invasive mechanical ventilation at baseline). A more detailed discussion of the results of all RCTs included in the present systematic review is available in the Supplementary Material. Another RCT was excluded as conducted only in mechanically ventilated patients [135].

Despite the limitations reported above (e.g., conflicting results with regard to methylprednisolone and hydrocortisone administration), a key aim of our literature search was that of trying to define a threshold of severity for differentiating non-invasively ventilated patients in which the intervention (steroid administration) was associated with better clinically relevant outcomes from non-invasively ventilated patients in which there was no favorable (and possibly an unfavorable) effect of steroid administration. However, it turned out that such a threshold could not be defined, based on 
currently available evidence from RCTs, for the following major reasons: (1) the lack of details regarding the type of non-invasive respiratory support in the RECOVERY trial; (2) the lack of information about viral clearance, which could have allowed an additional stratification for assessing efficacy in subgroups independent (or in combination) with the level of respiratory support; (3) the above reported conflicting results of the RCTs addressing the efficacy of steroids other than dexamethasone, which still deserve further investigation.

With the same aim (defining a threshold of disease severity to guide a targeted steroid administration in non-invasively ventilated patients) we also reviewed the observational literature on this subject, with a focus on the following relevant clinical outcomes: (1) survival; (2) need for invasive mechanical ventilation; (3) progression to severe disease. However, it rapidly became clear that no clear conclusion could be drawn. The major factors hampering a clear definition of a threshold of severity from observational studies were (1) the various definitions of severe COVID-19 pneumonia or disease used in the different studies (i.e., subgroups hardly comparable between studies); (2) the different steroid drugs and dosages used in the different studies; (3) the non-homogeneous definitions of primary outcome measures.

\section{Conclusive Remarks}

In available RCTs, a general trend towards improved survival was consistently observed with dexamethasone administration in critically ill patients undergoing invasive mechanical ventilation, whereas for inpatients with COVID-19 not undergoing invasive mechanical ventilation the evidence is less straightforward. Certainly, the positive effect of dexamethasone in inpatients with COVID-19 requiring supplemental oxygen (and of methylprednisolone in those aged 60 years or older), cannot be ignored. The evidence in support of these effects is less solid, based on post hoc subgroup analyses, but, in the opinion of the panel, sufficient to justify the use of these drugs in these populations. However, it must be underlined that these recommendations will likely need to be refined in the future according to a more precise stratification based on the type of non-invasive respiratory support (e.g., nasal cannula, Venturi mask, continuous positive airway pressure [CPAP], non-invasive ventilation [NIV]), and on other patients' characteristics (e.g., viral load) that may be considered in the risk/benefit balance of steroid administration. Finally, as the related evidence stemmed from RCTs conducted in ICUs, albeit also in non-invasively ventilated patients, the panel decided not to provide recommendations about the possible use of hydrocortisone at the present time, pending further evidence that could be extrapolated to inpatients with COVID-19 outside ICU.

\section{Recommendations*}

- Unless contraindicated, we recommend the use of dexamethasone at the dosage of $6 \mathrm{mg} /$ day for 10 days in inpatients with COVID-19 requiring oxygen supplementation ** -weak recommendation, low certainty of evidence

- Methylprednisolone at the dosage of $0.5 \mathrm{mg} /$ $\mathrm{kg}$ twice daily for at least 5 days could be considered in inpatients with COVID-19 requiring oxygen supplementation and aged 60 years or older-weak recommendation, very low certainty of evidence

*These recommendations are intended for inpatients with COVID-19 outside ICU.

${ }^{* *}$ Equivalent dosages of other steroids may be considered if dexamethasone is not available (although this should be considered as best practice recommendation, taking also into account the indirectness of evidence for steroids other than dexamethasone).

\section{Future Research Directions}

- To assess whether a threshold of severity guided by well-defined types of oxygen supplementation and other clinical/laboratory parameters may differentiate with better accuracy non-invasively ventilated inpatients with COVID-19 who benefit from steroid administration from those who do not

- To compare in randomized studies the efficacy in terms of relevant clinical outcomes 
of different steroids and of different steroid dosages

- To assess in follow-up studies, preferably randomized, whether steroids administration (and their different formulations/dosages) may help to prevent or reduce the possible development of nonreversible pulmonary fibrosis in hospitalized patients with moderate/severe COVID-19 pneumonia

\section{Question 5: Should Antiviral Agents be Administered to Inpatients with COVID- 19 ?}

\section{Evidence Summary}

The possibility of exerting direct antiviral activity against the virus has prompted several studies to be conducted, including a non-negligible number of RCTs assessing the efficacy and safety of either already existing or novel drugs with direct antiviral activity for the treatment of patients with COVID-19. For the purpose of guidelines development, we focused the discussion of the available evidence on the results of RCTs assessing the efficacy of antiviral drugs compared to standard care alone (with/or without placebo) in hospitalized patients with COVID-19 not subjected to invasive mechanical ventilation (as the population assessed by the present question, for the same reasons detailed in question 4), with respect to the following clinically relevant endpoints: (1) mortality; (2) need for invasive mechanical ventilation; (3) clinical improvement or recovery. In the following paragraphs we summarize the major indications stemming from RCTs for guiding panel recommendations on the possible use of antivirals in the target population. A more detailed report of the results of RCTs included in the present review is available in the Supplementary Material.

Lopinavir/Ritonavir Lopinavir/ritonavir (LPV/ r) is a combination of drugs to treat human immunodeficiency virus (HIV) infection. LPV is a protease inhibitor, whereas ritonavir is a booster increasing the plasma half-life of the former. In in vitro studies, LPV was shown to inhibit a protease crucial for coronavirus replication and conserved in SARS-CoV-2 [136, 137]. Based on these premises and on some encouraging results from animal models of SARS-CoV2 infection [138], in the first phase of the pandemic LPV/r was administered to several patients with COVID-19, either within an RCT or as an off-label treatment [4]. Subsequently, after some observational experiences had provided conflicting preliminary results about any possible favorable effect of $\mathrm{LPV} / \mathrm{r}$ in patients with COVID-19 [139, 140], results from RCTs started to become available. In an open-label RCT conducted in China in hospitalized patients with COVID-19, treatment with LPV/r was compared with standard care alone [141] and was not found to be associated with a shorter time to clinical improvement (primary study endpoint), or with reduced mortality (secondary endpoint). This lack of effect was confirmed in two subsequent, open-label RCTs (the RECOVERY RCT and the WHO-sponsored SOLIDARITY RCT) [142, 143]. In both studies, most patients were not under mechanical ventilation at baseline, and mortality was the primary endpoint. Based on the results of these three RCTs, and despite some limitations (e.g., all the three RCTs were open-label and a higher baseline throat viral loads was registered in the $\mathrm{LPV} / \mathrm{r}$ arm in one of the studies [141]), the panel believes that the consistent lack of effect registered for LPV/r in randomized studies does not support its use in hospitalized patients with COVID-19.

Remdesivir Remdesivir is a nucleotide analogue with broad antiviral activity that was developed against Ebola virus. Remdesivir showed in vitro activity against coronaviruses [144], including SARS-CoV-2 [145], and possible favorable effects were also supported by animal studies [146]. Before emergency/conditional approval by US and European regulatory entities, during the first months of the pandemic remdesivir was administered to hospitalized patients with COVID-19 within compassionate use programs. The aforementioned approvals were then granted on the basis of the first concluded RCTs evaluating the efficacy of remdesivir in hospitalized patients with COVID-19. In 
the Adaptive Covid-19 Treatment Trial (ACTT1), a double-blind RCT assessing the efficacy of remdesivir (10-day course) in hospitalized patients with COVID-19 and evidence of lower pulmonary tract infection [147], the primary endpoint was time to recovery, which was ultimately shorter in the remdesivir than in the placebo arm (in a population in which more than $70 \%$ of patients were not under mechanical ventilation at baseline). There was no evidence that the positive effect of remdesivir was less marked/absent in patients who were not under mechanical ventilation at baseline (the target population of the present recommendation), in whom, on the contrary, this effect was apparently more marked, especially in those receiving low-flow oxygen supplementation.

With regard to possible different dosages of remdesivir, in an open-label RCT involving 397 hospitalized patients with COVID-19 not requiring invasive mechanical ventilation at enrollment, a 5-day course and a 10-day course of remdesivir (both with a dose of $200 \mathrm{mg}$ the first day and then $100 \mathrm{mg} /$ day) showed comparable clinical improvement at day 14 as the primary efficacy endpoint [148]. In another open-label RCT conducted in hospitalized patients with moderate COVID-19 pneumonia [149], clinical improvement at day 11 was observed in $70 \%(134 / 191), 65 \%$ (126/193), and $61 \%(121 / 200)$ of patients in the 5-day remdesivir, 10-day remdesivir, and standard care arms, respectively. The results of another doubleblind RCT investigating the use of 10-day remdesivir in hospitalized patients with COVID-19 pneumonia were less favorable than those of the ACTT-1 study, with remdesivir being not associated with reduced time to clinical improvement, and with similar 28-day mortality being observed in remdesivir and placebo arms [150]. In addition, the results of an interim analysis of the WHO-sponsored SOLIDARITY RCT (in which less than 10\% of patients were under mechanical ventilation at baseline and the primary endpoint was in-hospital mortality) also showed similar mortality in remdesivir-treated and placebo-treated patients (13\% in both arms) [143]. Notably, the SOLIDARITY trial also included a meta-analysis of all the RCTs including remdesivir reported above (with the exception of the one comparing two different dosages, without standard care arm), with respect to the mortality endpoint, showing a summary rate ratio (RR) of 0.91 (95\% CI 0.79-1.05) vs. standard care as reference. Although with the inherent limitations of subgroup analyses, in this meta-analysis a trend towards reduced mortality in remdesivir arms was observed across RCTs in the subgroups of patients not receiving mechanical ventilation, while an opposite trend towards increased mortality was observed in the subgroups of patients receiving mechanical ventilation (in the SOLIDARITY RCT) and invasive mechanical ventilation (in the ACTT-1 RCT). Complete results of the SOLIDARITY trial will help to further (and possibly ultimately) clarify this point. Indeed, while any possible positive effect of remdesivir in invasively ventilated patients is very unlikely according to the available data from the RCTs, in non-invasively ventilated patients a possible favorable effect in terms of reduced mortality cannot be ruled out at the present time. For this reason, the panel currently supports the use of remdesivir in noninvasively ventilated patients with COVID-19 requiring oxygen supplementation, recognizing that this recommendation is not based on high certainty of evidence.

Hydroxychloroquine Hydroxychloroquine (HCQ) and chloroquine are aminoquinolines drugs well established as treatment for rheumatic diseases and malaria. The proposed use of these drugs for the treatment of patients with COVID-19 was based both on their possible immunomodulatory effects and on their possible antiviral activity [3], with the latter also explaining its presence in this section of evidence synthesis. At the beginning of the COVID-19 pandemic, many hopes were put on HCQ (which has a more favorable toxic profile than chloroquine), as a consequence of its in vitro activity against SARS-CoV-2 [145] and the hypothesis-generating results of a small observational, retrospective study showing a possible favorable effect of HCQ in terms of reduced SARS-CoV-2 viral load in respiratory specimens [151]. Conflicting observational results showing a possible lack of favorable 
effect of HCQ on different endpoints were subsequently provided by other exploratory observational studies [140, 152-154]. For all these reasons, results of RCTs were largely awaited to ultimately either support or discourage the use of HCQ in patients with COVID-19. Available evidence from RCTs about the use of HCQ in outpatients has been already discussed (see question 2). Here we focus on its use in inpatients. With the exception of a small open-label RCT conducted in Egypt among 194 hospitalized patients with COVID-19 not undergoing invasive mechanical ventilation at enrollment [155], in which recovery within 28 days was achieved in 53.6\% (52/97) and 34.0\% (33/97) of patients in HCQ and standard care arms, respectively, results from larger RCT suggested against substantial efficacy of HCQ in hospitalized patients with COVID-19. In an open-label RCT conducted in hospitalized patients with mild to moderate COVID-19 [64], no beneficial effect in terms of proportional odds of having a higher clinical score at day 15 (primary endpoint) was found for HCQ compared to standard care (OR 1.21, 95\% CI 0.69-2.11) and for azithromycin plus HCQ compared to standard care (OR 0.99, 95\% CI 0.57-1.73). In the RECOVERY RCT [156], the primary endpoint of 28-day mortality was registered in 27\% (421/ $1561)$ and $25 \%(790 / 3155)$ of patients randomized to HCQ and standard care arms, respectively (RR 1.09, 95\% CI 0.97-1.23). In an interim analysis of the open-label SOLIDARITY RCT [143], in-hospital death (primary endpoint) occurred in 11.0\% (104/947) and 9.3\% (84/906) of patients in the HCQ and standard care arms, respectively (RR 1.19, 95\% CI 0.89-1.59). Of note, in all these RCTs the proportion of patients receiving invasive mechanical ventilation at baseline was limited, and the observed efficacy results were consistent in the larger subgroups of patients not ventilated at baseline. The RCTs discussed above also did not show a favorable effect of HCQ in terms of secondary endpoints of major clinical interest such as mortality or progression to mechanical ventilation (see Supplementary Material for more details). Almost all subsequent RCTs (and all the large ones) were in line with these findings, suggesting no substantial effect of HCQ administration in hospitalized patients with COVID-19 [157-163]. Of note, the results of at least two meta-analyses supported the lack of effect of HCQ in reducing short-term mortality in patients hospitalized with COVID-19, although mostly based on observational studies $[164,165]$. Finally, three RCTs did not investigate clinical outcomes (mortality, need for mechanical ventilation, disease progression/ improvement), but qualitative or quantitative changes in viral load (negative conversion of SARS-CoV-2 molecular tests on respiratory specimen by day 28 , reduction of viral load in nasopharyngeal swabs by day 7 , or decline in viral load by day 4) as the primary endpoints $[57,166,167]$. No substantial effects of HCQ administration vs. standard care alone with respect to these virological primary endpoints were found in these trials $[57,166,167]$.

Despite some limitations that should be recognized (such as the different dosages and duration of HCQ employed in the various RCTs [168]), in the opinion of the panel the consistent lack of effect registered in the large RCTs included in the present review do not support the use HCQ in the target population according to current evidence.

Other Antiviral Agents Although other antiviral agents such as umifenovir, favipiravir, interferons, leflunomide, sofosbuvir, and daclatasvir have been proposed for the treatment of inpatients with COVID-19, there is currently no solid supporting evidence from large RCTs. For this reason, the panel currently do not recommend their use for treating patients with COVID-19 outside RCTs. More details regarding in vitro evidence and results of exploratory observational studies and preliminary RCTs are available in the Supplementary Material.

\section{Conclusive Remarks}

At the beginning of the COVID-19 pandemic, there was great expectation that antiviral agents, based on promising results of in vitro and exploratory observational studies, could significantly improve the outcome of patients with COVID-19. However, evidence provided by RCTs has mainly indicated a lack of effect, 
ultimately leading the panel to discourage the use of $\mathrm{LPV} / \mathrm{r}$ and $\mathrm{HCQ}$, while retaining a recommendation for the use remdesivir, in which a limited (but possible) favorable effect on survival in the target population cannot be currently excluded. Nonetheless, although the initial expectations were not fully met, this certainly does not mean that efforts to find other, efficacious antiviral treatments should be discontinued, especially considering the need to enrich our knowledge about any possible, optimized combined use of antiviral plus antiinflammatory and/or immunomodulatory agents.

\section{Recommendations *}

- LPV/r should not be administered to hospitalized patients with COVID-19-strong recommendation, moderate certainty of evidence

- Pending further results from large RCTs, administration of a 5-day course of remdesivir should be considered in hospitalized patients with COVID-19 pneumonia requiring oxygen supplementation-weak recommendation, very low certainty of evidence

- HCQ should not be administered to hospitalized patients with COVID-19—strong recommendation, moderate certainty of evidence

- Other antiviral agents should not be administered for treating COVID-19 in hospitalized patients, unless they are administered within RCTs - best practice recommendation (based on expert opinion only)

*These recommendations are intended for inpatients with COVID-19 outside ICU.

\section{Future Research Directions}

- To provide further and definitive results regarding the efficacy of remdesivir in hospitalized patients with COVID-19 not requiring invasive mechanical ventilation

- To provide results from large RCTs regarding the efficacy of antiviral agents other than $\mathrm{LPV} / \mathrm{r}$, remdesivir, and HCQ with respect to clinically relevant endpoints in hospitalized patients with COVID-19 not requiring invasive mechanical ventilation

- To provide results from large RCTs regarding the efficacy of combination of antiviral agents or between antiviral and immunomodulatory agents with respect to clinically relevant endpoints in hospitalized patients with COVID-19 not requiring invasive mechanical ventilation

Question 6: Should Antibiotics be Administered to Inpatients with COVID19 ?

\section{Evidence Summary}

Besides their mandatory use in proven bacterial infections, antibiotics are mostly administered to hospitalized patients with COVID-19 not requiring invasive mechanical ventilation in the following two situations: (1) at hospital admission, in patients with suspected pulmonary bacterial co-infection or superinfection; (2) for the empirical treatment, pending microbiological results, of severe hospital-acquired bacterial superinfections occurring later during hospital stay. At least in general, the administration of antibiotics in this latter situation may follow the common rules and clinical reasoning for the empirical treatment of hospital-acquired infections. Therefore, the main question of the present section refers mostly to the former situation (i.e., suspicion of mixed viral/bacterial pneumonia at hospital admission): in fact, it remains unclear whether or not to administer antibiotics to patients with COVID-19 presenting with consolidative infiltrates at chest X-ray/computerized tomography and with increased laboratory inflammatory markers (e.g., white blood cell count, C-reactive protein) that may resemble bacterial disease. Overall, this may lead to an indiscriminate and perilous use of antibiotics in many patients with COVID-19 without bacterial infections, likely increasing the selective pressure for antimicrobial resistance both in patients and in the environment. For this reason, we focused the first part of our evidence evaluation on the prevalence of bacterial pneumonia in hospitalized patients with COVID-19, preferably at hospital admission, and reviewed systematic reviews and meta-analyses of observational studies. In a systematic review of 30 observational studies for a total of 3834 hospitalized 
patients with COVID-19, the pooled prevalence of laboratory-confirmed bacterial pulmonary co-infection was 7\% (95\% CI 3-12, with high heterogeneity, $I^{2}=92.2 \%$ ) [169]. When excluding studies conducted exclusively in ICU, the pooled prevalence of bacterial co-infection was lower $(4 \%$, with $95 \%$ CI $1-9 \%$, still with high heterogeneity, $I^{2}=91.7 \%$ ). Of note, the term co-infection in this systematic review apparently included both co-infection and superinfections, and both community-acquired and hospital-acquired infections. The most commonly detected organism was Mycoplasma pneumoniae, followed by Pseudomonas aeruginosa. In another systematic review, including nine observational studies for a total of 806 hospitalized patients with COVID-19 (including both ICU and non-ICU patients with no clear distinction), 64 developed a bacterial or fungal infection (no differentiation between fungal and bacterial infections was presented nor was a pooled estimate of the prevalence provided) [170].

Despite such a low prevalence of bacterial infections, the same meta-analyses registered a high frequency of antibiotics administration to hospitalized patients with COVID-19 (more than 70\%), which seems not to be justified $[169,170]$. Yet, the presence of a beneficial effect would theoretically support widespread antibiotic administration even in the presence of a low prevalence of bacterial diseases. Therefore, as the second part of our evidence evaluation, we searched the literature with the aim of assessing the effect of antibiotic administration (as the intervention) in terms of relevant clinical outcomes (mortality, need for invasive mechanical ventilation) in hospitalized patients with COVID-19 not requiring invasive mechanical ventilation (main population). In addition, we aimed to identify subgroups in which the intervention (antibiotic administration) was most effective. However, identification of such subgroups was ultimately not possible (see "Effect of Antibiotics Administration"). Of note, in our assessment we were able to focus only on patient-level effects of antibiotic administration. Indeed, an important limitation was (and still is) that any possible longterm consequence at population-level of widespread antibiotic therapy cannot be evaluated at the present time, owing to the only recent spread of SARS-CoV-2.

Effect of Antibiotics Administration The most studied antibiotic for the treatment of patients with COVID-19 is azithromycin, but not for its antibacterial activity, rather for its possible antiviral/anti-inflammatory activity. Its use in outpatients with COVID-19 has been discussed in a previous section. In two RCTs conducted in hospitalized patients, the addition of azithromycin or azithromycin plus hydroxychloroquine to standard care was not associated with improved survival in hospitalized patients with COVID-19 [64, 171]. A large multicenter observational retrospective study provided similar results [153]. A lack of effect of azithromycin vs. placebo was also observed in the RECOVERY RCT, in which 2582 patients were randomized to receive azithromycin and 5181 patients to receive standard care alone, and the primary endpoint of 28-day mortality was registered in $22 \%$ of patients in both arms [172].

With regard to administration of antibiotics in general, 13 observational, retrospective studies were identified that explored the effect of antibiotics on relevant clinical outcomes (mortality in 12 studies and a composite of death, ICU admission, and need for mechanical ventilation in 1 study) in hospitalized patients with COVID-19 [173-185]. It is worth noting that it was not possible to separate the results observed in ICU and non-ICU patients, and in patients receiving invasive mechanical ventilation or not requiring it. The proportion of invasively ventilated patients ranged from $6.2 \%$ to $33.0 \%$ in the different studies. In none of the studies was antibiotic administration associated with an increased survival, although the following points should be highlighted: (1) all were univariable or descriptive comparisons, with no multivariable models including other potentially prognostic covariates, beside antibiotic administration; (2) the proportion of patients receiving antibiotics was consistently greater than $80 \%$ in all studies and thus the number of patients not receiving antibiotics was small; (3) negative confounding may be expected because of an increased use of antibiotics in 
worsening patients not responding to supportive care for COVID-19. No extrapolation of results for any subgroup was possible in the included studies. Finally, it should be noted that, despite the wide literature search, some papers addressing the impact of antibiotics on relevant clinical outcomes may have been missed (as antibiotics were usually not the main focus of many studies in which they were only one among several factors tested for association with clinical outcomes).

For a more detailed description of the pertinent results of the studies included in this section (i.e., those evaluating the effect of azithromycin or antibiotics in general on relevant clinical outcomes) see the Supplementary Material.

\section{Conclusive Remarks}

With the exception of azithromycin, there is currently no high-level evidence either against or in favor of a widespread administration of antibiotics to all hospitalized patients with COVID-19. However, the low prevalence of bacterial co-infections registered in systematic reviews of observational studies could be more in line with a parsimonious use of antibiotics, thereby discouraging their routine use in the target population. Notably, no information is currently available about the possible effect of antibiotics in subgroups stratified according to specific clinical or laboratory variables; thus further research is needed to develop dedicated prediction models of bacterial infection in hospitalized patients with COVID-19 not requiring invasive mechanical ventilation; such models could help in identifying subgroups of patients in whom to administer empirical antibiotics for suspected bacterial pneumonia.

\section{Recommendations*}

- We recommend against the routine use of antibiotics in hospitalized patients with COVID-19 without proven bacterial infection-strong recommendation, moderate certainty of evidence (for azithromycin); weak recommendation, very low certainty of evidence (for antibiotics in general)
- We recommend collection of respiratory specimens for culture or molecular detection of respiratory pathogens, blood cultures, and urinary antigens for Streptococcus pneumoniae and Legionella spp. in hospitalized patients with COVID-19 and suspected bacterial pneumonia-best practice recommendation (based on expert opinion only)

- Empirical antibiotic treatment of suspected bacterial pneumonia alongside proper diagnostic procedures should be considered in patients with COVID-19 with evidence of consolidative radiological lesions-best practice recommendation (based on expert opinion only)

- In the case of empirical antibiotic treatment, selection of agents to be administered should follow standard practice for the treatment of bacterial pneumonia-best practice recommendation (based on expert opinion only)

*These recommendations are intended for inpatients with COVID-19 outside ICU.

\section{Future Research Directions}

- To develop dedicated prediction models of bacterial infection in hospitalized patients with COVID-19 that could help in shaping preferential subgroups of patients in whom to administer empirical antibiotics

- To identify the optimal diagnostic approach (including also the role of laboratory markers of infection/inflammation and rapid microbiological tests) to bacterial infections in hospitalized patients with COVID-19

\section{Question 7: Should Neutralizing Monoclonal Antibodies and Non-Steroid Immunomodulators be Administered to Inpatients with COVID-19?}

\section{Evidence Summary}

Since the first published reports of clinical manifestations and laboratory findings in hospitalized patients with COVID-19, it was postulated that two different components contributed to the organ damage: (1) the virus; (2) a dysregulated inflammatory host response to the virus [186-188]. 
From an immunological perspective, different agents may act on these two components: (1) by neutralizing key viral antigens; (2) by reducing an excessive inflammatory host response. Regarding point (2), several anti-inflammatory or immunomodulatory agents, besides steroids, were administered as off-label treatments in many hospitalized patients with COVID-19 during the first months of the pandemic, supported by observational studies of various sizes and quality, indicating a possible favorable effect [189-192]. Subsequently, the results of RCTs assessing the effect of some nonsteroid anti-inflammatory and/or immunomodulatory agents on relevant clinical outcomes (mortality, need for invasive mechanical ventilation/ICU admission, clinical improvement) in hospitalized patients with COVID-19 became available. These RCTs, together with those on neutralizing monoclonal antibodies (whereas convalescent plasma is specifically addressed in question 8 ), were the focus of the literature search for the present question and are discussed in the following paragraphs. As for other questions, the population addressed in the present question was inpatients not requiring invasive mechanical ventilation, owing to the stratification according to the type of ventilatory support adopted by many RCTs.

Neutralizing Monoclonal Antibodies The main target of SARS-CoV-2 neutralizing monoclonal antibodies is the surface spike glycoprotein that mediates viral entry into host cells, and positive results from RCTs are available for the treatment of outpatients with COVID-19 (see question 2). Bamlanivimab and the combination of casirivimab and imdevimab have been also evaluated for the treatment of hospitalized patients with COVID-19. Results from RCTs in hospitalized patients currently seem less favorable than in outpatients, although some analyses are still ongoing.

In a preplanned interim analysis of the TICO platform RCT (which is contemporaneously evaluating different treatments for COVID-19), the comparison between LY-CoV555 plus remdesivir and with placebo plus remdesivir met the prespecified criteria for futility in hospitalized patients with COVID-19 (no patient was under invasive mechanical ventilation at enrollment) [193]. For this reason, randomization to LY-CoV555 was halted at the end of October 2020. More details are reported in the Supplementary Material.

In an RCT assessing efficacy of REGN-COV2 in hospitalized patients, inpatients with COVID-19 were randomized to REGN-COV2 or placebo in four different, independent strata: (1) patients not requiring oxygen; (2) patients on low-flow oxygen; (3) patients on high-flow oxygen; (4) patients on mechanical ventilation [194]. In October 2020, an independent data and safety monitoring board (DSMB) recommended halting continuation of the study in patients requiring high-flow oxygen or mechanical ventilation because of a potential safety signal and an unfavorable risk/benefit profile. The study is ongoing in the remaining groups (patients not requiring oxygen and patients on low-flow oxygen) and full results are still to be released.

Interleukin-6 Inhibitors Tocilizumab is a monoclonal antibody that blocks interleukin-6 (IL-6), a proinflammatory cytokine that has been hypothesized to contribute to the dysregulated response to the virus leading to lung and other organ damage. Since the beginning of the pandemic, several exploratory, observational, comparative studies suggested a possible benefit of tocilizumab administration over standard care alone in patients with COVID-19, including also the population of interest for the present question (hospitalized patients with COVID-19 not requiring invasive mechanical ventilation) [195-201]. While some of these observational studies were well-designed, large multicenter efforts with careful adjustment for potential confounding factors, they can provide only preliminary evidence, still burdened by residual and unobserved confounding. Although the presence of favorable results in observational studies was taken somewhat into consideration, we mostly focused our literature search and provided the pertinent recommendations on the basis of the results of currently available randomized studies. 
Overall, seven RCTs (three double-blind and four open-label) were initially retrieved by our search that assessed the efficacy of tocilizumab in hospitalized patients with COVID-19 not requiring invasive mechanical ventilation at baseline and included completely or in part non-ICU patients [202-208]. Their results with regard to the respective primary endpoints (which varied across studies, ranging from clinical improvement on an ordinal scale to a composite of death, mechanical ventilation, and clinical worsening) were somewhat conflicting. In the RCT by Salvarani and colleagues, who enrolled hospitalized patients receiving oxygen through high-flow nasal cannula or Venturi mask, a lack of effect with respect to the primary composite endpoint of ICU admission, death, or clinical worsening led to enrollment discontinuation for futility, whereas in the study by Hermine and colleagues (enrolling hospitalized patients requiring low-flow oxygen supplementation) a favorable effect on one of the primary efficacy endpoints (survival without need for non-invasive/invasive mechanical ventilation by day 14) was achieved [202, 204]. The trial by Salama and colleagues, conducted in hospitalized patients with COVID-19 not requiring invasive mechanical ventilation, showed an advantage of tocilizumab in terms of the primary composite endpoint of mechanical ventilation or death by day 28 , whereas the one by Stone and colleagues, conducted in hospitalized patients with moderate COVID-19, did not show advantages of tocilizumab with respect to the primary efficacy endpoint (composite of intubation or death) [203, 205]. A composite of intubation or death was also assessed in the open-label RCT by Veiga and colleagues, in which hospitalized patient with severe of critical COVID-19 (16\% were under mechanical ventilation at baseline) were randomized to receive tocilizumab or standard of care alone, showing no benefit of tocilizumab administration [208]. The RCT was halted prematurely because of an increased number of deaths in the tocilizumab arm at day $15(17 \%$ vs. 3\%). The COVACTA RCT by Rosas and colleagues, conducted in hospitalized patients with severe COVID-19 pneumonia (of whom 38\% were in ICU at enrollment), showed no advantages vs. placebo in terms of the primary endpoint of improved clinical status at day 28 [206]. In the open-label RCT by Soin and colleagues, conducted in hospitalized patients with moderate to severe COVID-19 (of whom 5\% were under mechanical ventilation), no substantial difference was observed with respect to the primary endpoint of disease progression between patients receiving tocilizumab or standard care alone [207].

A detailed description of the methods and relevant results of each of these trials is available in the Supplementary Material. The inconsistency in the results of these RCTs is not easily explained (although differences in the characteristics of baseline population and presence/ absence of ceiling respiratory treatments may play a role [209]) and precludes any solid conclusion on the efficacy of tocilizumab in patients with COVID-19 at the present time. It is also worth noting that none of the seven RCTs initially retrieved by our search reported an advantage of tocilizumab in terms of mortality either as a primary or a secondary endpoint, and that the pooled results do not suggest the presence of any worthwhile mortality advantage (see Fig. S2 in the Supplementary Material). All together, these considerations initially led the panel not to recommend the use of tocilizumab outside ongoing RCTs in the target population of these guidelines. However, the following two additional considerations should be made regarding RCTs on tocilizumab: (1) the results of the RECOVERY RCT related to the comparison of tocilizumab vs. standard care alone in 4116 hospitalized patients (of whom $14.1 \%$ in ICU) with progression of COVID-19 (defined as oxygen saturation $<92 \%$ on room air or supplementary oxygen therapy, and serum C-reactive protein $\geq 75 \mathrm{mg} / \mathrm{L}$ ) have been recently published, showing a favorable effect of tocilizumab vs. standard care alone with respect to the primary endpoint of 28-day mortality, that were consistent in pre-planned subgroup analyses, in particular with a major effect being detected in patients already receiving steroids [210]; further details are available in the Supplementary Material and Fig. S2; (2) the very recent favorable efficacy results regarding tocilizumab or sarilumab (another IL-6- 
mitigating agent) administration of the REMAPCAP trial were also partly considered by the panel for the present recommendations, although they refer to ICU patients and not to the target population of the present guidelines [211]. Of note, in this RCT a positive effect was retained in post hoc subgroup analyses in patients not receiving invasive mechanical ventilation (i.e., those ICU patients under noninvasive mechanical ventilation or high-flow nasal cannula) either for the primary endpoint of respiratory and cardiovascular organ supportfree days and for hospital survival [211].

Other agents that mitigate the effects of IL-6, including the already discussed sarilumab and siltuximab, are also under evaluation for the treatment of patients with COVID-19. Recently, the results of a phase 3 RCT assessing efficacy of sarilumab in hospitalized patients with COVID19 requiring oxygen supplementation were published, showing no effect on the primary endpoint of clinical improvement on a 7-point ordinal scale [212].

\section{Other Non-steroid Immunomodula-}

tors Anakinra is a recombinant IL-1 receptor antagonist. In a multicenter, Bayesian, openlabel RCT conducted in hospitalized patients with mild-to-moderate COVID-19 pneumonia, administration of anakinra in addition to standard care did not result in improved critical outcomes in comparison to standard care alone (see the Supplementary Material for more details) [213].

Baricitinib is a selective inhibitor of Janus kinase (JAK) 1 and 2 that inhibits the intracellular signaling pathway of cytokines known to be elevated in severe COVID-19 (IL-2, IL-6, IL10 , interferon- $\gamma$, and granulocyte-macrophage colony-stimulating factor). Recently, the results of a double-blind RCT evaluating baricitinib plus remdesivir vs. placebo plus remdesivir in hospitalized adults with COVID-19 were published [214, 215]. Of note, patients receiving steroids for the treatment of COVID-19 were excluded. In this trial, baricitinib plus remdesivir was superior to remdesivir alone in terms of improved median time to recovery, especially in patients receiving high-flow oxygen or noninvasive ventilation in subgroup analyses. Fewer adverse events were also observed in the baricitinib plus remdesivir arm. A more detailed description of the pertinent results of this RCT is available in the Supplementary Material.

Several other biologic immunomodulators have been used off-label and/or are being investigated in hospitalized patients with COVID-19. There have been a number of small case series or small exploratory RCTs, which are not listed here. No data from large RCTs have been published or made available through press releases at the time of the present literature review, but several RCTs are ongoing. Of note, after some possibly encouraging preliminary results from a small RCT [216], the results of the large RECOVERY RCT investigating the efficacy of colchicine in hospitalized patients with COVID-19 were recently released in a pre-print form, and, if confirmed after peer-review, do not support its use [217].

\section{Conclusive Remarks}

At the current time, available evidence dictates against recommendation of neutralizing monoclonal antibodies in hospitalized patients with COVID-19, at least pending the results of the REGN-COV-2 RCT in patients not requiring oxygen and patients on low-flow oxygen.

In view of the conflicting results pertaining to primary endpoints and the apparent lack of effect on mortality, the panel initially decided against recommending tocilizumab in the target population. However, based on the favorable results of the RECOVERY RCT results, the panel revised the decision, posing a conditional recommendation to consider the administration of tocilizumab in patients not responding to steroid treatment and with oxygen saturation $<92 \%$ on room air (including those already on supplementary oxygen) and with increased inflammatory markers in the absence of proven/suspected bacterial or fungal infection. The panel nonetheless recognize that this recommendation is not based on high certainty of evidence, considering the non-univocal results of the various RCTs (see above).

In view of reported favorable effects in an RCT and pending further results from other randomized studies, the panel did not recommend against the use of baricitinib in 
hospitalized patients with COVID-19, provided it is authorized for this indication by the pertinent regulatory agencies.

Considering the current lack of data from large RCTs for other immunomodulatory agents, they cannot be currently recommended for the treatment of hospitalized patients with COVID-19. Nonetheless, since several RCTs are ongoing, they may lead to future modifications of the current recommendations should their results be favorable.

\section{Recommendations*}

- Pending further results from RCTs, we recommend against the administration of neutralizing monoclonal antibodies in hospitalized patients with COVID-19-strong recommendation, moderate certainty of evidence

- We recommend considering tocilizumab administration in hospitalized patients with COVID-19 not responding to steroid treatment, with oxygen saturation $<92 \%$ on room air (including those already on supplementary oxygen), and with increased inflammatory markers ${ }^{* *}$ in the absence of a proven or suspected bacterial or fungal infec-

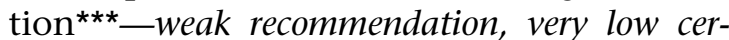
tainty of evidence

- Pending further results from RCTs, baricitinib may be considered in addition to remdesivir in patients requiring high-flow oxygen or non-invasive mechanical ventilation who are not under steroid treatment (e.g., in the presence of contraindications to steroid use)-weak recommendation, low certainty of evidence

- Pending further results from large RCTs, we recommend against administration of other non-steroid immunomodulatory agents outside RCTs-weak recommendation, very low certainty of evidence (for anakinra); best practice recommendation for other agents (based on expert opinion only)

*These recommendations are intended for inpatients with COVID-19 outside ICU.

**In the RECOVERY trial, serum C-reactive protein $\geq 75 \mathrm{mg} / \mathrm{L}$.

${ }^{* * *}$ Clinicians should be aware of the following: (1) the $75 \mathrm{mg} / \mathrm{L}$ cutoff is based on results of the RECOVERY RCT; (2) other markers of inflammation may be considered on a case-bycase basis (best practice recommendation); (3) another best practice recommendation is to avoid tocilizumab administration in patients with severe immunosuppression or in those with other contraindications to tocilizumab administration (low platelet count; risk of gastrointestinal perforation; increase of transaminases greater than five times the upper limit of normal).

\section{Future Research Directions}

- To provide further results from RCTs on the role of non-steroid immunomodulatory agents in hospitalized patients with COVID19 , in order to clarify the current conflicting evidence

- To improve our understanding on the role of inflammatory laboratory markers in defining subgroups/phenotypes that may maximize any possible favorable effect of non-steroid immunomodulatory agents in hospitalized patients with COVID-19

\section{Question 8: Should Convalescent Plasma be Administered to Inpatients with COVID-19?}

\section{Evidence Summary}

The plasma of patients recovering from COVID19 (convalescent plasma) may contain polyclonal antibodies that could neutralize viral antigens and have a beneficial effect if administered to hospitalized patients with COVID-19 [218]. The term hyperimmune immunoglobulin preparation refers to purified antibodies from convalescent plasma [219]. Several observational studies or single-arm interventional trials have been published regarding the use of convalescent plasma in patients with COVID-19 during the first months of the pandemic, with some promising results [220-236]. However, results from RCTs are now available, on which we focused our literature search, since they are able to provide higher certainty of evidence compared to observational and single-arm studies. Therefore, in the following paragraph we summarize the results of RCTs assessing 
efficacy in terms of relevant clinical outcomes (mortality, need for mechanical ventilation, and/or clinical improvement) of convalescent plasma or hyperimmune immunoglobulin for the treatment of hospitalized patients with COVID-19 not requiring invasive mechanical ventilation (population assessed in the present question instead of non-ICU patients according to the stratification according to ventilatory support in major RCT). Of note, results from pre-print (i.e., yet to be peer-reviewed) RCTs are also discussed and were taken into account for drafting recommendations. As for other sections of the guidelines, pre-print results were not considered high-certainty evidence because of the lack of peer-review. Nonetheless, they remain useful for supporting (or casting doubts about) the direction of the effect registered in peer-reviewed studies.

Efficacy Results from RCTs In our search, we identified six published RCTs, of which three were conducted exclusively in the population of non-invasively ventilated patients, one also included patients requiring invasive mechanical ventilation at baseline (although in less than $30 \%$ of the entire study population), and one was conducted in geriatric institutions (transformed into low-complexity inpatients units) and in outpatients [237-242]. Their results with regard to the different primary endpoints (in two cases clinical improvement, in one a composite of disease progression and mortality, in one mortality, in one requirement of ventilation, and in one development of severe respiratory disease) are summarized in Table S2 in the Supplementary Material. A more detailed description of the different studies is also available in the Supplementary Material, which also includes the results of still to be peer-reviewed RCTs published on pre-print servers [243-248]. Finally, it is of note that in the largest published RCT, the RECOVERY RCT (randomized, controlled, open-label, adaptive platform trial comparing a range of possible treatments in hospitalized patients with COVID-19), there was no benefit of convalescent plasma administration when compared to standard care alone: the analysis was based on 11,558 randomized patients with the primary endpoint of 28 -day mortality being $24 \%$ both in convalescent plasma and standard care arms, respectively; RR 1.00, 95\% CI 0.93-1.07 [242]. Based on these results, enrollment to convalescent plasma in the RECOVERY trial was halted. As shown in Table $\mathrm{S} 2$ in the Supplementary Material, in most of the studies the administration of convalescent plasma did not result in superior outcomes, with the exception of the study by Libster and colleagues [240], in which the administration of convalescent plasma with high IgG titers in older adult patients within $72 \mathrm{~h}$ after the onset of mild COVID-19 symptoms was associated with a reduced risk of developing severe respiratory disease, and possibly of the study by Li and colleagues (potentially underpowered because of early termination) [238]. Overall, in all peer-reviewed RCTs no substantial effects were observed with respect to the secondary endpoint of mortality (Fig. S3 in the Supplementary Material, summarizing the impact of convalescent plasma on mortality in the four published studies and in pre-print manuscripts).

\section{Conclusive Remarks}

Available results from RCTs have failed to show a substantial advantage in terms of efficacy of convalescent plasma administration in the population of interest, although the favorable dose-dependent IgG effect after convalescent plasma infusions in older adults observed by Libster and colleagues delineates a possible place in therapy in specific situations, to be confirmed by further RCTs [240]. In our opinion, the current evidence on the possible efficacy of convalescent plasma remains unconvincing and controversial (as also confirmed by the different position of various organizations and societies regarding its emergency use [249]), regardless of any favorable safety result (large data from the expanded access program have shown a low incidence of adverse events related to transfusion and no evidence of disease enhancement $[218,220,225])$. On this basis, the panel would recommend further research on this topic, with the use of convalescent plasma to be currently reserved for patients enrolled in RCTs. In fact, several reasons, including the current lack of 
standards about the optimal dose (in terms of antibody titers) to be transfused, the aforementioned inconsistent results of clinical trials, and the lack of efficacy information about hyperimmune immunoglobulin preparations (RCTs are ongoing), do not allow one to rule out the possibility of an advantage under specific conditions of use and/or in certain patient subgroups, which still deserves further investigation. Of note, should a favorable effect be demonstrated in ongoing or future RCTs, any possible widespread use of convalescent plasma may be hampered by the difficulties in its collection and preparation, particularly during other possible, future exponential increases in the virus diffusion.

\section{Recommendations *}

- Pending further results from RCTs, currently we do not support the administration of convalescent plasma in hospitalized patients with COVID-19 outside RCTs-weak recommendation, low certainty of evidence

- Pending further results from RCTs, currently we do not support the administration of anti-COVID-19 hyperimmune immunoglobulin preparations in hospitalized patients with COVID-19 outside RCTs-best practice recommendation (based on expert opinion only)

* These recommendations are intended for inpatients with COVID-19 outside ICU.

\section{Future Research Directions}

- To provide results from RCTs regarding efficacy of hyperimmune immunoglobulin preparations in hospitalized patients with COVID-19

- To clarify whether specific subgroups of hospitalized patients with COVID-19 may benefit from convalescent plasma administration
Question 9: Should Continuous Positive Airway Pressure (CPAP)/Non-invasive Ventilation (NIV) be Employed for Treating Inpatients with COVID-19 with Acute Hypoxemic Respiratory Failure?

\section{Evidence Summary}

Non-invasive ventilation (NIV, which delivers a differential air pressure during inspiration and expiration) or continuous positive airway pressure (CPAP, which delivers a constant air pressure during inspiration and expiration) are widely used to treat patients with COVID-19 pneumonia with hypoxic respiratory failure and/or respiratory distress that do not respond to standard or high-flow oxygen supplementation, for the following reasons: (1) when intubation is still not indicated (thereby possibly avoiding it in the case of favorable response); (2) in patients with "do not intubate" (DNI) orders; (3) in unfortunate situations where the number of patients requiring invasive mechanical ventilation exceeds the availability of devices to provide it.

In all these situations, NIV and CPAP are used with the intention to improve patient outcomes, but no evidence on the efficacy of CPAP/NIV for the treatment of patients with COVID-19 with acute hypoxemic respiratory failure could be extrapolated from the studies retrieved in our literature review because of the lack of RCTs or high-quality comparative observational studies. In studies employing CPAP or NIV, all patients with acute respiratory failure not responding to standard oxygen supplementation generally receive NIV/CPAP, and a controlled study in which a proportion of patients is denied either procedure would be considered ethically unacceptable.

For this reason, we decided to assess the available descriptive evidence in the population of patients receiving NIV/CPAP. More specifically, we retrieved information about the proportions of NIV/CPAP patients who (1) eventually required intubation; (2) died before or after intubation. Certainly, these descriptive, indirect findings are of questionable validity and do not provide evidence in the absence of a control group. However, either as the treatment 
of choice or as ceiling treatment, in our opinion, an acceptable frequency of successful administration of CPAP/NIV (survival and cure without intubation) may still provide useful insights on any potential advantage of the use of these techniques.

Finally, owing to the wide heterogeneity in indications/uses and populations of patients with COVID-19 undergoing high-flow nasal oxygen (HNFO) in observational studies, the panel deemed it currently unfeasible to develop guidance for the use of HFNO in inpatients with COVID-19. Thus, this point was not addressed in the present review (but with the commitment to consider it in future guideline updates). Very importantly, any future assessment of the role of HFNO should consider the recently published results of the HENICOV RCT, comparing NIV vs. HFNO with respect to the primary outcome of days free of respiratory support at 28 days. Of note, no difference was observed in days free of respiratory support (20 vs.18 days for NIV and HFNO, respectively), although a lower number of intubation events and a higher number of invasive mechanical ventilation-free days were observed in the NIV arm [250, 251].

Overall, 25 studies were included, all of them observational (see Table S3 in the Supplementary Material for a schematic summary and the extended evidence summary in the Supplementary Material for further details on included studies) [252-276]. Mortality in patients treated with CPAP ranged from 14-24\% $[252,256,264,265,268,271]$ to $43-55 \%$ [254, 260], although possibly reaching $84 \%$ when CPAP was the ceiling treatment [276]. Mortality in patients treated with NIV was reported in five studies and ranged from 5\% to $52 \%$ (but reaching $86 \%$ in DNI patients) [261, 263, 269, 272, 274]. Pooling outcomes for the CPAP and NIV groups, Avdeev and colleagues estimated a mortality of $23 \%$ [255], and Bellani and colleagues estimated a mortality of 25\% [270], while pooled results from Burns et al. reported a 50\% mortality among DNI patients [257]. With regard to intubation (and thus need for invasive mechanical ventilation), it was observed in $41-63 \%$ of patients treated with CPAP [253, 254, 265, 273], but in some cases the proportion was as low as $11 \%$ [256]. In the study by Avdeev and colleagues, in which outcomes for patients treated with CPAP and those treated with NIV were pooled, intubation was eventually considered necessary in $28 \%$ of patients requiring $\mathrm{CPAP} / \mathrm{NIV}$, whereas intubation occurred in $15 \%$ of patients requiring CPAP/NIV in the study by Bellani and colleagues $[255,270]$. This heterogeneity of results can be attributed to different reasons: (1) the small sample size of several studies, which implies large statistical uncertainty in the estimates of mortality/intubation rates; (2) the various criteria for starting $\mathrm{CPAP} / \mathrm{NIV}$ adopted in the different studies; (3) the possible inclusion of DNI patients in the denominator; (4) difference in the instrumentation used and in pressure settings; (5) the different settings (general wards, high-dependency respiratory unit [HDRU], or ICU) in which the patients received NIV/CPAP. With regard to the last point, it is worth noting that for the present question we did not exclude studies conducted exclusively in ICU, as patients were inherently not receiving invasive mechanical ventilation at baseline (although the questionable generalizability of these results to non-ICU settings was taken into account when developing recommendations). A more detailed description of all the aforementioned sources of heterogeneity in the different studies is provided in the Supplementary Material, which also reports the available, limited results concerning direct comparisons of CPAP vs. NIV, which seem to indicate similar mortality, although the analyses are frequently unadjusted and possibly biased. With regard to safety of CPAP/NIV (which was rarely assessed in included studies), Aliberti and coworkers reported a low prevalence of pneumothorax/pneumomediastinum in patients exposed to CPAP treatment (1.9\%) [252], while Franco and colleagues reported none [259]. Tolerance to CPAP was generally under-reported, with available data on proportion of CPAP interruption ranging from $0 \%$ to $44 \%$ [264, 268]. No information could be extrapolated from included studies regarding safety of NIV. 


\section{Conclusive Remarks}

All the studies that assessed clinical outcomes in hospitalized patients with COVID-19 with acute respiratory failure treated with CPAP and/or NIV have an observational design, and the great majority are based on single-center data. The studies included in the analysis demonstrated a significant heterogeneity in patients' characteristics and clinical presentation, especially with regard to the severity of respiratory failure, DNI reporting, and criteria for CPAP/NIV initiation. Moreover, the instrumentation used and the pressure settings are reported only in a minority of cases. The lack of these data contributes to the difficult interpretation of the results and limits the validity and generalizability of the findings. Finally, characteristics and outcomes of patients exposed to NIV and CPAP are sometimes pooled, implying similarities between these non-invasive approaches. However, CPAP and NIV represent very different ventilatory support modalities, needing different instrumentation and settings. Differences in mortality and intubation rates vary consistently from study to study and can be only partially explained by different respiratory failure severity at baseline or any possible different proportion of elderly or DNI patients in the sample enrolled.

Given these limitations which preclude a recommendation based on GRADE criteria, it is nonetheless the opinion of the panel, based on the proportions of survival in NIV/CPAP patients (also when used as ceiling treatment) reported in the different studies, despite their heterogeneity, that CPAP and NIV are feasible approaches in patients showing respiratory distress, persistent respiratory failure, or inadequate oxygenation despite standard supplementation. Complications, such as pneumothorax and pneumomediastinum, seems to be limited, and in the majority of cases likely caused by excessive positive end-expiratory pressure (PEEP). In the opinion of the panel, it would be reasonable not to exceed a PEEP of $10 \mathrm{cmH}_{2} \mathrm{O}$, unless differently indicated by the pulmonary/critical care physician.

Considering the widespread utilization of non-invasive ventilatory approaches in patients with persistent hypoxemia and COVID-19 pneumonia, there is an urgent need for welldesigned RCTs that would guide patients' selection and suggest the best management in clinical practice.

Hopefully, results of several RCTs that are currently enrolling patients or activating participating centers (e.g., NCT04381923, NCT04326075, NCT04390191, NCT04715243) could eventually help to precisely define the place in therapy of CPAP and NIV in standardized therapeutic algorithms for patients with COVID-19.

\section{Recommendations *}

- Unless contraindicated, non-invasive ventilatory support by means of NIV or CPAP is feasible and safe in patients with acute respiratory failure secondary to COVID-19, and should be considered for patients in whom standard oxygen supplementation is not or no longer sufficient and who do not require immediate intubation-best practice recommendation (based on expert opinion only)

- CPAP delivery systems allowing for PEEP titration should be preferred, and PEEP should not exceed $10 \mathrm{cmH}_{2} \mathrm{O}$-best practice recommendation (based on expert opinion only)

*These recommendations are intended for inpatients with COVID-19 outside ICU.

\section{Future Research Directions}

- To assess efficacy and safety of CPAP/NIV in patients with COVID-19 with acute hypoxemic respiratory failure in RCTs

- To define standardized criteria for initiation/ use of CPAP and NIV in patients with COVID-19, for both clinical and research purposes

- To assess in prospective studies, preferably RCTs, possible differences in terms of clinically relevant outcomes of CPAP vs. NIV when employed in patients with acute respiratory failure secondary to COVID-19 
Question 10: When Can an Improved Patient with COVID-19 be Discharged from an Acute Care Hospital?

\section{Evidence Discussion}

Decisions about discharge of a patient with COVID-19 from an acute care hospital, once their condition has improved, imply two different but related considerations: (1) From a clinical standpoint, is the patient sufficiently improved to be discharged? (2) From a public health standpoint is the patient still contagious?

To properly answer these two questions, in the opinion of the panel the following specifications are required: (1) population of interest is represented by hospitalized patients with COVID-19; (2) the intervention under study is discharge from acute care hospitals, and (3) the optimal outcome is a balanced composite of clinically relevant outcomes, quality of life, and healthcare costs (the last point also implies effects of possible transmission of the virus if the patient is still contagious, thereby creating an overlap between the two questions). Through our systematic literature review, we did not find articles assessing the effect of hospital discharge in these terms that would have allowed us to confidently identify specific subgroups or characteristics of patients to optimize the decisions (and the timing) of discharge.

Considering this lack of evidence, which precluded the possibility of defining recommendations through the GRADE system, we ultimately based our recommendations on experts' opinions. In this regard, in the official documents from national and international organizations there is substantial consistency about the opportunity to combine evidence of viral RNA clearance from the upper respiratory tract with the clinical improvement/resolution of symptoms for guiding discharge decisions [277-279]. However, the panel underlines that this combination must be evaluated on a caseby-case basis, since contagiousness may become irrelevant for discharge decisions if the patient can adopt isolation at home or is discharged to a long-term care facility able to guarantee isolation. In our opinion, this moves the central question from "When can a patient with
COVID-19 be discharged?" to "How can we reduce the excess in-hospital stay in patients with COVID-19 who still need to be isolated according to Italian regulations (see Table 2) but who no longer need hospitalization because of improved clinical conditions?" In our opinion, a proactive, organized collaboration between hospitals, long-term care facilities, and dedicated community facilities (for those isolated patients that could be discharged home but cannot adopt isolation) remains crucial for guaranteeing an adequate turnover in overcrowded hospitals and reducing any possible delay in the care of other patients with COVID19 needing hospitalization.

\section{Conclusive Remarks}

The literature search for this question did not provide any evidence satisfying the agreed rigorous selection criteria (and thus providing evidence of sufficient quality to guide definition of recommendations based on GRADE criteria). In the opinion of the panel, from a clinical standpoint, a patient with COVID-19 may be discharged from acute care hospitals when oxygen supplementation is no longer required or with a maximum requirement of low-flow oxygen at $2 \mathrm{~L} / \mathrm{min}$ through nasal cannula (with the exception of patients already under oxygen supplementation at home at baseline or patients requiring initiation of long-term oxygen therapy after discharge), in line with common practice with other types of noncontagious lower respiratory tract infections, and provided there are no complications or other medical reasons that require continuation of hospitalization. For patients with COVID-19 still requiring isolation but who could be discharged from a clinical standpoint, isolation outside the hospital (at home, in community facilities, or in long-term facilities, according to the specific need for non-acute care of any given patient) should be supported and made feasible for as many patients as possible, especially in the case of overcrowded hospitals.

\section{Recommendations}

- Clinically stable patients with COVID-19 who no longer require isolation (or who can 
Table 2 Discontinuation of isolation in patients with COVID-19 according to the Italian Ministry of Health

\section{Type of hospitalized patient with COVID- Indication for discontinuing isolation}

19

Previously symptomatic patient

The patient can be de-isolated after at least 10 days from the onset of symptoms, provided the patient had a negative molecular test for SARSCoV-2 performed after at least 10 days from the onset of symptoms and after at least 3 days from disappearance of symptoms (with the exception of anosmia and ageusia/dysgeusia that may last longer)

Previously symptomatic patient with persistent positivity of molecular tests
The patient can be de-isolated after at least 21 days from the onset of symptoms even in the presence of persistent positivity of molecular tests, after at least 7 days from disappearance of symptoms (with the exception of anosmia and ageusia/dysgeusia that may last longer). This criterion may be modulated by health authorities in accordance with experts, with special attention to the immune status of patients (in immunocompromised patients the shedding of viable viral particles may be prolonged)

Adapted from [279]

be isolated outside the hospital) should be discharged from acute care hospitals when oxygen supplementation is no longer required or with a maximum requirement of low-flow oxygen at $2 \mathrm{~L} / \mathrm{min}$ through nasal cannula (with the exception of patients already under oxygen supplementation at home at baseline or patients requiring initiation of long-term oxygen therapy after discharge), in line with common practice with other types of non-contagious lower respiratory tract infections, and provided there are no complications or other reasons that require continuation of hospitalization-best practice recommendation (based on expert opinion only)

- For patients with COVID-19 still requiring isolation but who could be discharged from a clinical standpoint, isolation outside the hospital (at home, in community facilities, or in long-term facilities, according to the specific need for non-acute care of any given patient) should be supported and made feasible for as many patients as possiblebest practice recommendation (based on expert opinion only)

\section{Future Research Directions}

- To assess the cost-effectiveness of discharge decisions in patients with COVID-19 (and in subgroups according to different baseline characteristics and disease courses) by balancing clinically relevant outcomes (both short-term and long-term), quality of life, and healthcare costs

\section{CONCLUSIONS}

The present first version of the SITA and SIP guidelines on the clinical management of adult patients with COVID-19 outside ICUs systematically addressed ten different relevant questions, aiming to improve dissemination of a detailed, structured summary of the current evidence. As detailed in the various sections, future versions of this guidance document should refine or modify current recommendations on the basis on novel, high-quality evidence, as well as address other crucial aspects of the clinical approach to patients with COVID19 outside ICUs, including the appropriate prevention and management of the long-term effects of the disease. 


\section{ACKNOWLEDGEMENTS}

Funding. No funding or sponsorship was received for this study or publication of this article.

Authorship. All named authors meet the International Committee of Medical Journal Editors (ICMJE) criteria for authorship for this article, take responsibility for the integrity of the work as a whole, and have given their approval for this version to be published.

Author Contributions. Matteo Bassetti: project chair for SITA, project concept, voting panel member, revision of final manuscript and supplementary material. Francesco Blasi: project chair for SIP, project concept, voting panel member, revision of final manuscript and supplementary material. Daniele Roberto Giacobbe: project coordinator, methodology and systematic reviews, assessment of evidence with the GRADE system, drafting of recommendations, drafting of final manuscript and supplementary material, supervision of voting process. Pierluigi Viale, Malgorzata Mikulska, Nicola Petrosillo, Andrea Gori, Carlo Tascini, Francesco Giuseppe De Rosa, Pierachille Santus, Fabiano Di Marco, Stefano Centanni, Carlo Vancheri, Angelo Gratarola, Federico Pea: voting panel members, revision of final manuscript and supplementary material. Antonio Vena, Guido Granata, Silvia Corcione, Emanuela Sozio, Nadia Castaldo, Andrea Lombardi, Andrea Gramegna, Dejan Radovanovic, Elena Tagliabue: search strings development, conduction of systematic reviews, drafting of recommendations, revision of final manuscript and supplementary material. Alessio Signori: development and supervision of methodology together with the project coordinator, revision of final manuscript and supplementary material. Alberto Enrico Maraolo: development and supervision of methodology together with the project coordinator, assessment of evidence with the GRADE system, search strings development, revision of final manuscript and supplementary material. Paolo Bruzzi: development and supervision of methodology together with the project coordinator, assessment of evidence with the GRADE system, revision of final manuscript and supplementary material.

List of Investigators. Matteo Bassetti, Daniele Roberto Giacobbe, Paolo Bruzzi, Emanuela Barisione, Stefano Centanni, Nadia Castaldo, Silvia Corcione, Francesco Giuseppe De Rosa, Fabiano Di Marco, Andrea Gori, Andrea Gramegna, Guido Granata, Angelo Gratarola, Alberto Enrico Maraolo, Malgorzata Mikulska, Andrea Lombardi, Federico Pea, Nicola Petrosillo, Dejan Radovanovic, Pierachille Santus, Alessio Signori, Emanuela Sozio, Elena Tagliabue, Carlo Tascini, Carlo Vancheri, Antonio Vena, Pierluigi Viale, Francesco Blasi.

Disclosure. Outside the submitted work, Daniele Roberto Giacobbe reports unconditional grants from MSD Italia, Correvio Italia, and Pfizer Inc. Outside the submitted work, Matteo Bassetti has received funding for scientific advisory boards, travel and speaker honoraria from Angelini, Astellas, Bayer, BioMérieux, Cidara, Cipla, Gilead, Menarini, MSD, Pfizer, Shionogi, Tetraphase, Nabriva. Outside the submitted work, Francesco Blasi reports grants and personal fees from AstraZeneca, grants from Bayer, grants and personal fees from Chiesi, grants and personal fees from GlaxoSmithKline, personal fees from Grifols, personal fees from Guidotti, personal fees from Insmed, grants and personal fees from Menarini, personal fees from Novartis, grants and personal fees from Pfizer, personal fees from Zambon, and personal fees from Vertex. Outside the submitted work, Emanuela Barisione reports personal fees from Boehringer Ingelheim, personal fees from Chiesi, and personal fees from GlaxoSmithKline. Outside the submitted work, Federico Pea participated in speaker bureau for Angelini, Basilea Pharmaceutica, Gilead, Hikma, Merck Sharp \& Dohme, Nordic Pharma, Pfzer and Sanofi Aventis, and in advisory board for Angelini, Basilea Pharmaceutica, Correvio, Gilead, Hikma, Merck Sharp \& Dohme, Nordic Pharma, Novartis, Pfizer, Shionogi and ThermoFisher. Outside the submitted work, Nicola Petrosillo reports personal fees from MSD, personal fees from Pfizer, personal fees from 
Johnson \& Johnson, personal fees from Shionogi, personal fees from Takeda, and personal fees from Becton \& Dickinson. Outside the submitted work, Pierachille Santus reports personal fees from Gilead. Paolo Bruzzi, Stefano Centanni, Nadia Castaldo, Silvia Corcione, Francesco Giuseppe De Rosa, Fabiano Di Marco, Andrea Gori, Andrea Gramegna, Guido Granata, Angelo Gratarola, Alberto Enrico Maraolo, Malgorzata Mikulska, Andrea Lombardi, Dejan Radovanovic, Alessio Signori, Emanuela Sozio, Elena Tagliabue, Carlo Tascini, Carlo Vancheri, Antonio Vena, and Pierluigi Viale have nothing to disclose.

Compliance with Ethics Guidelines. This article is based on previously conducted studies and does not contain any new studies with human participants or animals performed by any of the authors.

Guidelines. Springer Healthcare is not responsible for the validity of guidelines it publishes.

Open Access. This article is licensed under a Creative Commons Attribution-NonCommercial 4.0 International License, which permits any non-commercial use, sharing, adaptation, distribution and reproduction in any medium or format, as long as you give appropriate credit to the original author(s) and the source, provide a link to the Creative Commons licence, and indicate if changes were made. The images or other third party material in this article are included in the article's Creative Commons licence, unless indicated otherwise in a credit line to the material. If material is not included in the article's Creative Commons licence and your intended use is not permitted by statutory regulation or exceeds the permitted use, you will need to obtain permission directly from the copyright holder. To view a copy of this licence, visit http:// creativecommons.org/licenses/by-nc/4.0/.

\section{REFERENCES}

1. Ioannidis JPA. Global perspective of COVID-19 epidemiology for a full-cycle pandemic. Eur J Clin Invest. 2020;2020:e13423.

2. European Center for Disease Control and Prevention (ECDC). COVID-19 situation update worldwide, as of week 7, updated 25 February 2021. https://www.ecdc.europa.eu/en/geographicaldistribution-2019-ncov-cases. Accessed 27 February 2021.

3. Bassetti M, Giacobbe DR, Aliberti S, et al. Balancing evidence and frontline experience in the early phases of the COVID-19 pandemic: current position of the Italian Society of Anti-infective Therapy (SITA) and the Italian Society of Pulmonology (SIP). Clin Microbiol Infect. 2020;26:880-94.

4. Bassetti M, Pelosi P, Robba C, et al. A brief note on randomized controlled trials and compassionate/ off-label use of drugs in the early phases of the COVID-19 pandemic. Drugs Context. 2020;2020:9.

5. Zagury-Orly I, Schwartzstein RM. Covid-19-a reminder to reason. N Engl J Med. 2020;383:e12.

6. ESMO/European Sarcoma Network Working Group. Gastrointestinal stromal tumours: ESMO Clinical Practice Guidelines for diagnosis, treatment and follow-up. Ann Oncol. 2014; 25(Suppl3):iii21-26.

7. Tsuji BT, Pogue JM, Zavascki AP, et al. International consensus guidelines for the optimal use of the polymyxins: endorsed by the American College of Clinical Pharmacy (ACCP), European Society of Clinical Microbiology and Infectious Diseases (ESCMID), Infectious Diseases Society of America (IDSA), International Society for Anti-infective Pharmacology (ISAP), Society of Critical Care Medicine (SCCM), and Society of Infectious Diseases Pharmacists (SIDP). Pharmacotherapy. 2019;39: 10-39.

8. Guyatt GH, Oxman AD, Vist GE, et al. GRADE: an emerging consensus on rating quality of evidence and strength of recommendations. BMJ. 2008;336: 924-6.

9. Moher D, Liberati A, Tetzlaff J, et al. Preferred reporting items for systematic reviews and metaanalyses: the PRISMA statement. BMJ. 2009;339: b2535.

10. The Ottawa Hospital Research Institute. The Newcastle-Ottawa Scale (NOS) for assessing the quality of nonrandomised studies in meta-analyses. 2021. http://ohri.ca/programs/clinical_epidemiology/ oxford.asp. Accessed 28 Feb 2021. 
11. Cochrane Effective Practice and Organization of Care. Suggested risk of bias criteria for EPOC reviews. EPOC resources for review authors. Suggested risk of bias criteria for EPOC reviews. 2021. https://epoc.cochrane.org/sites/epoc.cochrane.org/ files/public/uploads/Resources-for-authors2017/ suggested_risk_of_bias_criteria_for_epoc_reviews. pdf. Accessed 28 Feb 2021.

12. Gottlieb M, Sansom S, Frankenberger C, et al. Clinical course and factors associated with hospitalization and critical illness among COVID-19 patients in Chicago. Illinois Acad Emerg Med. 2020;27:963-73.

13. Lighter J, Phillips M, Hochman S, et al. Obesity in patients younger than 60 years is a risk factor for COVID-19 hospital admission. Clin Infect Dis. 2020;71:896-7.

14. Petrilli CM, Jones SA, Yang J, et al. Factors associated with hospital admission and critical illness among 5279 people with coronavirus disease 2019 in New York City: prospective cohort study. BMJ. 2020;369:m1966.

15. Prieto-Alhambra D, Ballo E, Coma E, et al. Filling the gaps in the characterization of the clinical management of COVID-19: 30-day hospital admission and fatality rates in a cohort of 118150 cases diagnosed in outpatient settings in Spain. Int J Epidemiol. 2020;2020:5.

16. Soares RCM, Mattos LR, Raposo LM. Risk factors for hospitalization and mortality due to COVID-19 in Espirito Santo State. Brazil Am J Trop Med Hyg. 2020;103:1184-90.

17. Imam Z, Odish F, Armstrong J, et al. Independent correlates of hospitalization in 2040 patients with COVID-19 at a large hospital system in Michigan, Unites States. J Gen Intern Med. 2020;35:2516-7.

18. Jehi L, Ji X, Milinovich A, et al. Development and validation of a model for individualized prediction of hospitalization risk in 4,536 patients with COVID-19. PLoS ONE. 2020;15:e0237419.

19. Ko JY, Danielson ML, Town M, et al. Risk factors for COVID-19-associated hospitalization: COVID-19associated hospitalization surveillance network and behavioral risk factor surveillance system. Clin Infect Dis. 2020;2020:5.

20. Carrillo-Vega MF, Salinas-Escudero G, Garcia-Pena $\mathrm{C}$, et al. Early estimation of the risk factors for hospitalization and mortality by COVID-19 in Mexico. PLoS ONE. 2020;15:e0238905.

21. Fresan U, Guevara M, Elia, F et al. Independent role of severe obesity as a risk factor for COVID-19 hospitalization: a Spanish population-based cohort study. Obesity (Silver Spring). 2021;29(1):29-37.

22. Halalau A, Imam Z, Karabon P, et al. External validation of a clinical risk score to predict hospital admission and in-hospital mortality in COVID-19 patients. Ann Med. 2021;53:78-86.

23. Clift AK, Coupland CAC, Keogh RH, et al. Living risk prediction algorithm (QCOVID) for risk of hospital admission and mortality from coronavirus 19 in adults: national derivation and validation cohort study. BMJ. 2020;371:m3731.

24. Hao B, Sotudian S, Wang T, et al. Early prediction of level-of-care requirements in patients with COVID19. Elife. 2020;2020:9.

25. Izurieta HS, Graham DJ, Jiao Y, et al. Natural history of COVID-19: risk factors for hospitalizations and deaths among $>26$ million U.S. Medicare beneficiaries. J Infect Dis. 2021;29;223(6):945-56.

26. Prieto-Alhambra D, Ballo E, Coma E, et al. Filling the gaps in the characterization of the clinical management of COVID-19: 30-day hospital admission and fatality rates in a cohort of 118150 cases diagnosed in outpatient settings in Spain. Int J Epidemiol. 2021;49:1930-9.

27. Reilev M, Kristensen KB, Pottegard A, et al. Characteristics and predictors of hospitalization and death in the first 11122 cases with a positive RTPCR test for SARS-CoV-2 in Denmark: a nationwide cohort. Int J Epidemiol. 2020;49:1468-81.

28. Szente-Fonseca SN, de Queiroz-Sousa A, Wolkoff AG, et al. Risk of hospitalization for Covid-19 outpatients treated with various drug regimens in Brazil: comparative analysis. Travel Med Infect Dis. 2020;38:101906.

29. Shah BA, Ahmed W, Dhobi GN, et al. Validity of pneumonia severity index and CURB-65 severity scoring systems in community acquired pneumonia in an Indian setting. Indian J Chest Dis Allied Sci. 2010;52:9-17.

30. Satici C, Demirkol MA, Sargin Altunok E, et al. Performance of pneumonia severity index and CURB-65 in predicting 30-day mortality in patients with COVID-19. Int J Infect Dis. 2020;98:84-9.

31. Ji D, Zhang D, Xu J, et al. Prediction for progression risk in patients with COVID-19 pneumonia: the CALL score. Clin Infect Dis. 2020;71:1393-9.

32. Garcia-Clemente MM, Herrero-Huertas J, Fernandez-Fernandez A, et al. Assessment of risk scores in Covid-19. Int J Clin Pract. 2020;2020:e13705. 
33. Myrstad M, Ihle-Hansen $H$, Tveita AA, et al. National Early Warning Score 2 (NEWS2) on admission predicts severe disease and in-hospital mortality from Covid-19-a prospective cohort study. Scand J Trauma Resusc Emerg Med. 2020;28: 66.

34. Iijima Y, Okamoto T, Shirai T, et al. MuLBSTA score is a useful tool for predicting COVID-19 disease behavior. J Infect Chemother. 2020;2020:5.

35. Guo L, Wei D, Zhang X, et al. Clinical features predicting mortality risk in patients with viral pneumonia: the MuLBSTA score. Front Microbiol. 2019;10:2752.

36. Grifoni E, Valoriani A, Cei F, et al. The CALL score for predicting outcomes in patients with COVID-19. Clin Infect Dis. 2021;72:182-3.

37. Kostakis I, Smith GB, Prytherch D, et al. The performance of the National Early Warning Score and National Early Warning Score 2 in hospitalised patients infected by the severe acute respiratory syndrome coronavirus 2 (SARS-CoV-2). Resuscitation. 2021;159:150-7.

38. Sze S, Pan D, Williams CML, et al. Letter to the editor: variability but not admission or trends in NEWS2 score predicts clinical outcome in elderly hospitalised patients with COVID-19. J Infect. 2021;82:159-98.

39. Bello-Chavolla OY, Antonio-Villa NE, Ortiz-Brizuela $\mathrm{E}$, et al. Validation and repurposing of the MSLCOVID-19 score for prediction of severe COVID-19 using simple clinical predictors in a triage setting: The Nutri-CoV score. PLoS ONE. 2020;15:e0244051.

40. Bradley P, Frost F, Tharmaratnam K, et al. Utility of established prognostic scores in COVID-19 hospital admissions: multicentre prospective evaluation of CURB-65, NEWS2 and qSOFA. BMJ Open Respir Res. 2020;2020:7.

41. Cetinkal G, Kocas BB, Ser OS, et al. Assessment of the modified CHA2DS2VASc risk score in predicting mortality in patients hospitalized with COVID-19. Am J Cardiol. 2020;135:143-9.

42. Dorjee K, Kim H, Bonomo E, Dolma R. Prevalence and predictors of death and severe disease in patients hospitalized due to COVID-19: a comprehensive systematic review and meta-analysis of 77 studies and 38,000 patients. PLOS ONE. 2020;15: e0243191.

43. Foieni F, Sala G, Mognarelli JG, et al. Derivation and validation of the clinical prediction model for COVID-19. Intern Emerg Med. 2020;15:1409-14.
44. Guo L, Xiong W, Liu D, et al. The mNCP-SPI score predicting risk of severe COVID-19 among mildpneumonia patients on admission. Infect Drug Resist. 2020;13:3593-600.

45. Gupta RK, Marks M, Samuels THA, et al. Systematic evaluation and external validation of 22 prognostic models among hospitalised adults with COVID-19: an observational cohort study. Eur Respir J. 2020;2020:56.

46. Haimovich AD, Ravindra NG, Stoytchev S, et al. Development and validation of the quick COVID19 severity index: a prognostic tool for early clinical decompensation. Ann Emerg Med. 2020;76:442-53.

47. Hajifathalian K, Sharaiha RZ, Kumar S, et al. Development and external validation of a prediction risk model for short-term mortality among hospitalized US COVID-19 patients: a proposal for the COVID-AID risk tool. PLoS ONE. 2020;15: e0239536.

48. Lopez-Pais J, Otero DL, Ferreiro TG, et al. Fast track triage for COVID-19 based on a population study: the soda score. Prev Med Rep. 2021;21:101298.

49. Rodriguez-Nava G, Yanez-Bello MA, Trelles-Garcia DP, et al. Performance of the quick COVID-19 severity index and the Brescia-COVID respiratory severity scale in hospitalized patients with COVID19 in a community hospital setting. Int J Infect Dis. 2021;102:571-6.

50. Ruocco G, McCullough PA, Tecson KM, et al. Mortality risk assessment using CHA(2)DS(2)-VASc scores in patients hospitalized with coronavirus disease 2019 infection. Am J Cardiol. 2020;137: 111-7.

51. Shi Y, Pandita A, Hardesty A, et al. Validation of pneumonia prognostic scores in a statewide cohort of hospitalised patients with COVID-19. Int J Clin Pract. 2020;2020:e13926.

52. Torres-Macho J, Ryan P, Valencia J, et al. The PANDEMYC Score. An easily applicable and interpretable model for predicting mortality associated with COVID-19. J Clin Med. 2020;2020:9.

53. Vaughan L, Veruttipong D, Shaw JG, et al. Relationship of socio-demographics, comorbidities, symptoms and healthcare access with early COVID19 presentation and disease severity. BMC Infect Dis. 2021;21:40.

54. Italian Minister of Health. Agenzia Nazionale per i Servizi Sanitari Regionali (AGENAS). Criteri di appropriatezza per i setting assistenziali di gestione dei pazienti affetti da COVID-19. 2021. https:// www.agenas.gov.it/comunicazione/primo-piano/ 1837-criteri-di-appropriatezza-per-i-setting- 
assistenziali-di-gestione-dei-pazienti-affetti-dacovid-19. Accessed 27 May 2021.

55. Derwand R, Scholz M, Zelenko V. COVID-19 outpatients: early risk-stratified treatment with zinc plus low-dose hydroxychloroquine and azithromycin: a retrospective case series study. Int J Antimicrob Agents. 2020;56:106214.

56. Mikami T, Miyashita H, Yamada T, et al. Risk factors for mortality in patients with COVID-19 in New York City. J Gen Intern Med. 2021;36(1):17-26.

57. Mitja O, Corbacho-Monne $\mathrm{M}$, Ubals $\mathrm{M}$, et al. Hydroxychloroquine for early treatment of adults with mild Covid-19: a randomized-controlled trial. Clin Infect Dis. 2020; ciaa1009. https://doi.org/10. 1093/cid/ciaa1009.

58. Skipper CP, Pastick KA, Engen NW, et al. Hydroxychloroquine in nonhospitalized adults with early COVID-19: a randomized trial. Ann Intern Med. 2020;173:623-31.

59. Johnston C, Brown ER, Stewart J, et al. Hydroxychloroquine with or without azithromycin for treatment of early SARS-CoV-2 infection among high-risk outpatient adults: a randomized clinical trial. EClinicalMedicine. 2021;33:100773.

60. Reis G, Moreira Silva EADS, Medeiros Silva DC, et al. Effect of early treatment with hydroxychloroquine or lopinavir and ritonavir on risk of hospitalization among patients with COVID-19: the TOGETHER randomized clinical trial. JAMA Netw Open. 2021;4: e216468.

61. The RECOVERY Collaborative Group, Horby P, Lim WS, et al. Dexamethasone in hospitalized patients with Covid-19. N Engl J Med. 2021;384(8):693-704.

62. Ramakrishnan S, Nicolau DV Jr, Langford B, et al. Inhaled budesonide in the treatment of early COVID-19 (STOIC): a phase 2, open-label, randomised controlled trial. Lancet Respir Med. 2021;:2213-2600(21):00160.

63. Agusti A, Torres F, Faner R. Early treatment with inhaled budesonide to prevent clinical deterioration in patients with COVID-19. Lancet Respir Med. 2021;S2213-2600(21):00171-5.

64. Cavalcanti AB, Zampieri FG, Rosa RG, et al. Hydroxychloroquine with or without azithromycin in mild-to-moderate Covid-19. N Engl J Med. 2020;383:2041-52.

65. Butler CC, Dorward J, Yu L-M, et al. Azithromycin for community treatment of suspected COVID-19 in people at increased risk of an adverse clinical course in the UK (PRINCIPLE): a randomised, controlled, open-label, adaptive platform trial. Lancet. 2021;397(10279):1063-74.

66. Tardif J-C, Bouabdallaoui N, L'Allier PL, et al. Colchicine for community-treated patients with COVID-19 (COLCORONA): a phase 3, randomised, double-blinded, adaptive, placebo-controlled, multicentre trial. Lancet Respir Med. 2021. https://doi. org/10.1016/S2213-2600(21)00222-8.

67. Deftereos SG, Giannopoulos G, Vrachatis DA, et al. Effect of colchicine vs standard care on cardiac and inflammatory biomarkers and clinical outcomes in patients hospitalized with coronavirus disease 2019: the GRECCO-19 randomized clinical trial. JAMA Netw Open. 2020;3:e2013136.

68. Chen P, Nirula A, Heller B, et al. SARS-CoV-2 neutralizing antibody LY-CoV555 in outpatients with Covid-19. N Engl J Med. 2021;384(3):229-37.

69. Gottlieb RL, Nirula A, Chen P, et al. Effect of bamlanivimab as monotherapy or in combination with etesevimab on viral load in patients with mild to moderate COVID-19: a randomized clinical trial. JAMA. 2021;325:632-44.

70. Weinreich DM, Sivapalasingam S, Norton T, et al. REGN-COV2, a neutralizing antibody cocktail, in outpatients with Covid-19. N Engl J Med. 2021;384(3):238-51.

71. Agenzia Italiana del Farmaco (AIFA). Parere CTS AIFA su anticorpi monoclonali. Released on 4 Feb 2021. 2021. https://www.aifa.gov.it/documents/ 20142/1289678/parere_cts_monoclonali_04.02. 2021.pdf/68737075-6f07-2a43-7f94-0bc55f2e38f1. Accessed 27 May 2021.

72. Gonzalez-Ochoa AJ, Raffetto JD, Hernández AG, et al. Sulodexide in the treatment of patients with early stages of COVID-19: a randomized controlled trial. Thromb Haemost. 2021;121:944-54.

73. Caly L, Druce JD, Catton MG, et al. The FDA-approved drug ivermectin inhibits the replication of SARS-CoV-2 in vitro. Antiviral Res. 2020;178: 104787.

74. Chaccour C, Casellas A, Blanco-Di Matteo A, et al. The effect of early treatment with ivermectin on viral load, symptoms and humoral response in patients with non-severe COVID-19: a pilot, doubleblind, placebo-controlled, randomized clinical trial. EClinicalMedicine. 2021;32:100720.

75. López-Medina E, López P, Hurtado IC, et al. Effect of ivermectin on time to resolution of symptoms among adults with mild COVID-19: a randomized clinical trial. JAMA. 2021;325:1426-35. 
76. Mahmud R, Rahman MM, Alam I, et al. Ivermectin in combination with doxycycline for treating COVID-19 symptoms: a randomized trial. J Int Med Res. 2021;49:3000605211013550.

77. Chachar AZK, Khan KA, Asif M, et al. Effectiveness of ivermectin in SARS-CoV-2/COVID-19 patients. Int J Sci. 2021. https://doi.org/10.18483/ijSci.2378.

78. Podder CS, Chowdhury N, Sina MI, et al. Outcome of ivermectin treated mild to moderate COVID-19 cases: a single-centre, open-label, randomised controlled study. IMC J Med Sci. 2020;14:002.

79. Gu SX, Tyagi T, Jain K, et al. Thrombocytopathy and endotheliopathy: crucial contributors to COVID-19 thromboinflammation. Nat Rev Cardiol. 2021;18(3):194-209.

80. Della Bona R, Valbusa A, Malfa G, et al. Systemic fibrinolysis for acute pulmonary embolism complicating acute respiratory distress syndrome in severe COVID-19: a case series. Eur Heart J Cardiovasc Pharmacother. 2021;7(1):78-80.

81. Hunt BJ, De Paula EV, McLintock C, Dumantepe M. Prophylactic anticoagulation for patients in hospital with covid-19. BMJ. 2021;372:n487.

82. The ATTACC, ACTIV-4a, and REMAP-CAP Investigators, Lawler PR, Goligher EC, et al. Therapeutic anticoagulation in non-critically ill patients with Covid-19. medrxiv. 2021;2021.05.13.21256846. https://doi.org/10.1101/2021.05.13.21256846.

83. The REMAP-CAP, ACTIV-4a, ATTACC Investigators, Goligher EC, Bradbury CA, et al. Therapeutic anticoagulation in critically ill patients with Covid-19preliminary report. medRxiv. 2021; 2021.03.10. 21252749. https://doi.org/10.1101/2021.03.10. 21252749 .

84. INSPIRATION Investigators, Sadeghipour P, Talasaz $\mathrm{AH}$, et al. Effect of intermediate-dose vs standarddose prophylactic anticoagulation on thrombotic events, extracorporeal membrane oxygenation treatment, or mortality among patients with COVID-19 admitted to the intensive care unit: the INSPIRATION randomized clinical trial. JAMA. 2021;325:1620-1630.

85. Lopes RD, de Barros E Silva PGM, Furtado RHM, et al. Therapeutic versus prophylactic anticoagulation for patients admitted to hospital with COVID19 and elevated D-dimer concentration (ACTION): an open-label, multicentre, randomised, controlled trial. Lancet. 2021;397(10291):2253-2263

86. Albani F, Sepe L, Fusina F, et al. Thromboprophylaxis with enoxaparin is associated with a lower death rate in patients hospitalized with SARS-CoV-2 infection. A cohort study. EClinicalMedicine. 2020;27:100562.

87. Arslan Y, Yilmaz G, Dogan D, et al. The effectiveness of early anticoagulant treatment in Covid-19 patients. Phlebology. 2021;36(5):384-91.

88. Ayerbe L, Risco C, Ayis S. The association between treatment with heparin and survival in patients with Covid-19. J Thromb Thrombolysis. 2020;50: 298-301.

89. Billett HH, Reyes-Gil M, Szymanski J, et al. Anticoagulation in COVID-19: effect of enoxaparin, heparin, and apixaban on mortality. Thromb Haemost. 2020;120(12):1691-9.

90. Boari GEM, Chiarini G, Bonetti S, et al. Prognostic factors and predictors of outcome in patients with COVID-19 and related pneumonia: a retrospective cohort study. Biosci Rep. 2020;2020:40.

91. Bolzetta F, Maselli M, Formilan M, et al. Prophylactic or therapeutic doses of heparins for COVID19 infection? A retrospective study. Aging Clin Exp Res. 2021;33(1):213-17.

92. Desai A, Voza G, Paiardi S, et al. The role of antihypertensive treatment, comorbidities and early introduction of LMWH in the setting of COVID-19: a retrospective, observational study in Northern Italy. Int J Cardiol. 2021;324:249-54.

93. Elmelhat A, Elbourai E, Dewedar H, et al. Comparison between prophylactic versus therapeutic doses of low-molecular-weight heparin in severely ill coronavirus disease 2019 patients in relation to disease progression and outcome. Dubai Med J. 2020;2020:1-8.

94. Fauvel C, Weizman O, Trimaille A, et al. Pulmonary embolism in COVID-19 patients: a French multicentre cohort study. Eur Heart J. 2020;41:3058-68.

95. Ionescu F, Grasso-Knight G, Castillo E, et al. Therapeutic anticoagulation delays death in COVID-19 patients: cross-sectional analysis of a prospective cohort. TH Open. 2020;4:e263-70.

96. Lodigiani C, Iapichino G, Carenzo L, et al. Venous and arterial thromboembolic complications in COVID-19 patients admitted to an academic hospital in Milan. Italy Thromb Res. 2020;191:9-14.

97. Lynn L, Reyes JA, Hawkins K, et al. The effect of anticoagulation on clinical outcomes in novel Coronavirus (COVID-19) pneumonia in a US cohort. Thromb Res. 2021;197:65-68.

98. Paranjpe I, Fuster V, Lala A, et al. Association of treatment dose anticoagulation with in-hospital 
survival among hospitalized patients with COVID19. J Am Coll Cardiol. 2020;76:122-4.

99. Pesavento R, Ceccato D, Pasquetto G, et al. The hazard of (sub)therapeutic doses of anticoagulants in non-critically ill patients with Covid-19: the Padua province experience. J Thromb Haemost. 2020;18:2629-35.

100. Qin W, Dong F, Zhang Z, et al. Low molecular weight heparin and 28-day mortality among patients with coronavirus disease 2019: a cohort study in the early epidemic era. Thromb Res. 2020;198:19-22.

101. Russo V, Cardillo G, Viggiano GV, et al. Thromboprofilaxys with fondaparinux vs. enoxaparin in hospitalized COVID-19 patients: a multicenter Italian observational study. Front Med (Lausanne). 2020;7:569567.

102. Schiavone M, Gasperetti A, Mancone M, et al. Oral anticoagulation and clinical outcomes in COVID19: an Italian multicenter experience. Int J Cardiol. 2021;323:276-80.

103. Secco E, Pasqualetto MC, Bombardini T, et al. A possible benefit from therapeutic anticoagulation in patients with coronavirus disease 2019: the Dolo hospital experience in Veneto. Italy Kardiol Pol. 2020;78:919-21.

104. Tang N, Bai H, Chen X, et al. Anticoagulant treatment is associated with decreased mortality in severe coronavirus disease 2019 patients with coagulopathy. J Thromb Haemost. 2020;18:1094-9.

105. Dalager-Pedersen M, Lund LC, Mariager T, et al. Venous thromboembolism and major bleeding in patients with COVID-19: a nationwide populationbased cohort study. Clin Infect Dis. 2021; ciab003. https://doi.org/10.1093/cid/ciab003.

106. Di Castelnuovo AF, Costanzo S, Iacoviello L. Heparin in COVID-19 patients is associated with reduced in-hospital mortality: the multicentre Italian CORIST Study. Thromb Haemost. 2021. https:// doi.org/10.1055/a-1347-6070.

107. Ionescu F, Jaiyesimi I, Petrescu I, et al. Association of anticoagulation dose and survival in hospitalized COVID-19 patients: a retrospective propensity score-weighted analysis. Eur J Haematol. 2021;106: $165-74$.

108. Mouhat B, Besutti M, Bouiller K, et al. Elevated D-dimers and lack of anticoagulation predict PE in severe COVID-19 patients. Eur Respir J. 2020;2020: 56.
109. Musoke N, Lo KB, Albano J, et al. Anticoagulation and bleeding risk in patients with COVID-19. Thromb Res. 2020;196:227-30.

110. Saifi ES, Giorgi-Pierfranceschi M, Salvetti M, et al. Factors associated with survival in older patients affected by COVID-19: a retrospective cohort study. Arch Gerontol Geriatr. 2021;94:104349.

111. Shen L, Qiu L, Liu D, et al. The association of low molecular weight heparin use and in-hospital mortality among patients hospitalized with COVID-19. Cardiovasc Drugs Ther. 2021. https://doi.org/10. 1007/s10557-020-07133-3.

112. Arachchillage DRJ, Shi C, Saliu D, et al. Efficacy safety and of D-dimer, weight, and renal functionadjusted thromboprophylaxis in patients with coronavirus disease 2019 (COVID-19). Semin Thromb Hemost. 2021;47(4):436-41.

113. Tomasoni D, Inciardi RM, Lombardi CM, et al. Impact of heart failure on the clinical course and outcomes of patients hospitalized for COVID-19. Results of the Cardio-COVID-Italy multicentre study. Eur J Heart Fail. 2020;22:2238-47.

114. Canoglu K, Saylan B. Therapeutic dosing of lowmolecular-weight heparin may decrease mortality in patients with severe COVID-19 infection. Ann Saudi Med. 2020;40:462-8.

115. Hsu A, Liu Y, Zayac AS, et al. Intensity of anticoagulation and survival in patients hospitalized with COVID-19 pneumonia. Thromb Res. 2020;196: $375-8$.

116. Motta JK, Ogunnaike RO, Shah R, et al. Clinical outcomes with the use of prophylactic versus therapeutic anticoagulation in coronavirus disease 2019. Crit Care Explor. 2020;2:e0309.

117. Trimaille A, Curtiaud A, Marchandot B, et al. Venous thromboembolism in non-critically ill patients with COVID-19 infection. Thromb Res. 2020;193:166-9.

118. Prandoni P, Cattelan AM, Carrozzi L, et al. The hazard of fondaparinux in non-critically ill patients with COVID-19: retrospective controlled study versus enoxaparin. Thromb Res. 2020;196:395-7.

119. Bousquet G, Falgarone G, Deutsch D, et al. ADLdependency, D-Dimers, LDH and absence of anticoagulation are independently associated with onemonth mortality in older inpatients with Covid-19. Aging (Albany NY). 2020;12:11306-13.

120. Falcone M, Tiseo G, Barbieri G, et al. Role of lowmolecular-weight heparin in hospitalized patients with severe acute respiratory syndrome coronavirus 
2 pneumonia: a prospective observational study. Open Forum Infect Dis. 2020;7:ofaa563.

121. Russell CD, Millar JE, Baillie JK. Clinical evidence does not support corticosteroid treatment for 2019-nCoV lung injury. Lancet. 2020;395:473-5.

122. Shang L, Zhao J, Hu Y, et al. On the use of corticosteroids for 2019-nCoV pneumonia. Lancet. 2020;395:683-4.

123. Bartoletti M, Marconi L, Scudeller L, et al. Efficacy of corticosteroid treatment for hospitalized patients with severe COVID-19: a multicentre study. Clin Microbiol Infect. 2021;27(1):105-11.

124. Papamanoli A, Yoo J, Grewal P, et al. High-dose methylprednisolone in nonintubated patients with severe COVID-19 pneumonia. Eur J Clin Invest. 2020;2020:e13458.

125. Fatima SA, Asif M, Khan KA, et al. Comparison of efficacy of dexamethasone and methylprednisolone in moderate to severe covid 19 disease. Ann Med Surg (Lond). 2020;60:413-6.

126. Fernandez-Cruz A, Ruiz-Antoran B, Munoz-Gomez A, et al. A retrospective controlled cohort study of the impact of glucocorticoid treatment in SARSCoV-2 infection mortality. Antimicrob Agents Chemother. 2020;2020:64.

127. Monreal E, Sainz de la Maza S, Natera-Villalba E, et al. High versus standard doses of corticosteroids in severe COVID-19: a retrospective cohort study. Eur J Clin Microbiol Infect Dis. 2021;40(4):761-9.

128. Yuan M, Xu X, Xia D, et al. Effects of corticosteroid treatment for non-severe COVID-19 pneumonia: a propensity score-based analysis. Shock. 2020;54: 638-43.

129. Edalatifard M, Akhtari M, Salehi M, et al. Intravenous methylprednisolone pulse as a treatment for hospitalised severe COVID-19 patients: results from a randomised controlled clinical trial. Eur Respir J. 2020;56(6):2002808.

130. Jeronimo CMP, Farias MEL, Val FFA, et al. Methylprednisolone as adjunctive therapy for patients hospitalized with COVID-19 (Metcovid): a randomised, double-blind, phase IIb, placebo-controlled trial. Clin Infect Dis. 2021;72(9):e373-e381.

131. Corral-Gudino L, Bahamonde A, Arnaiz-Revillas F, et al. Methylprednisolone in adults hospitalized with COVID-19 pneumonia: an open-label randomized trial (GLUCOCOVID). Wien Klin Wochenschr. 2021;133:303-11.

132. Ranjbar K, Moghadami M, Mirahmadizadeh A, et al. Methylprednisolone or dexamethasone, which one is superior corticosteroid in the treatment of hospitalized COVID-19 patients: a triple-blinded randomized controlled trial. BMC Infect Dis. 2021;21: 337.

133. Angus DC, Derde L, Al-Beidh F, et al. Effect of hydrocortisone on mortality and organ support in patients with severe COVID-19: the REMAP-CAP COVID-19 corticosteroid domain randomized clinical trial. JAMA. 2020;324:1317-29.

134. Dequin PF, Heming N, Meziani F, et al. Effect of hydrocortisone on 21-day mortality or respiratory support among critically ill patients with COVID19: a randomized clinical trial. JAMA. 2020;324: 1298-306.

135. Tomazini BM, Maia IS, Cavalcanti AB, et al. Effect of dexamethasone on days alive and ventilator-free in patients with moderate or severe acute respiratory distress syndrome and COVID-19: the CoDEX randomized clinical trial. JAMA. 2020;324:1307-16.

136. Liu X, Wang XJ. Potential inhibitors against 2019-nCoV coronavirus M protease from clinically approved medicines. J Genet Genomics. 2020;47: 119-21.

137. Nukoolkarn V, Lee VS, Malaisree M, et al. Molecular dynamic simulations analysis of ritonavir and lopinavir as SARS-CoV 3CL(pro) inhibitors. J Theor Biol. 2008;254:861-7.

138. Park SJ, Yu KM, Kim YI, et al. Antiviral efficacies of FDA-approved drugs against SARS-CoV-2 infection in ferrets. MBio. 2020;2020:11.

139. Zuo Y, Liu Y, Zhong Q, et al. Lopinavir/ritonavir and interferon combination therapy may help shorten the duration of viral shedding in patients with COVID-19: a retrospective study in two designated hospitals in Anhui, China. J Med Virol. 2020;92:2666-74.

140. Choi MJ, Kang M, Shin SY, et al. Comparison of antiviral effect for mild-to-moderate COVID-19 cases between lopinavir/ritonavir versus hydroxychloroquine: a nationwide propensity score-matched cohort study. Int J Infect Dis. 2020;102: 275-81.

141. Cao B, Wang Y, Wen D, et al. A trial of lopinavirritonavir in adults hospitalized with severe Covid19. N Engl J Med. 2020;382:1787-99.

142. RECOVERY Collaborative Group. Lopinavir-ritonavir in patients admitted to hospital with COVID-19 (RECOVERY): a randomised, controlled, open-label, platform trial. Lancet. 2020;396(10259):1345-52.

143. WHO Solidarity Trial Consortium. Repurposed antiviral drugs for Covid-19-interim WHO 
solidarity trial results. N Engl J Med. 2021;384(6): 497-511.

144. Sheahan TP, Sims AC, Graham RL, et al. Broadspectrum antiviral GS-5734 inhibits both epidemic and zoonotic coronaviruses. Sci Transl Med. 2017;2017:9.

145. Wang M, Cao R, Zhang L, et al. Remdesivir and chloroquine effectively inhibit the recently emerged novel coronavirus (2019-nCoV) in vitro. Cell Res. 2020;30:269-71.

146. Williamson BN, Feldmann F, Schwarz B, et al. Clinical benefit of remdesivir in rhesus macaques infected with SARS-CoV-2. Nature. 2020;585:273-6.

147. Beigel JH, Tomashek KM, Dodd LE, et al. Remdesivir for the treatment of Covid-19_final report. N Engl J Med. 2020;383:1813-26.

148. Goldman JD, Lye DCB, Hui DS, et al. Remdesivir for 5 or 10 days in patients with severe Covid-19. N Engl J Med. 2020;383:1827-37.

149. Spinner CD, Gottlieb RL, Criner GJ, et al. Effect of remdesivir vs standard care on clinical status at 11 days in patients with moderate COVID-19: a randomized clinical trial. JAMA. 2020;324:1048-57.

150. Wang Y, Zhang D, Du G, et al. Remdesivir in adults with severe COVID-19: a randomised, double-blind, placebo-controlled, multicentre trial. Lancet. 2020;395:1569-78.

151. Gautret P, Lagier JC, Parola P, et al. Hydroxychloroquine and azithromycin as a treatment of COVID-19: results of an open-label non-randomized clinical trial. Int J Antimicrob Agents. 2020;56: 105949.

152. Geleris J, Sun Y, Platt J, et al. Observational study of hydroxychloroquine in hospitalized patients with Covid-19. N Engl J Med. 2020;382:2411-8.

153. Rosenberg ES, Dufort EM, Udo T, et al. Association of treatment with hydroxychloroquine or azithromycin with in-hospital mortality in patients with COVID-19 in New York State. JAMA. 2020;323: 2493-502.

154. Gao G, Wang A, Wang S, et al. Brief report: retrospective evaluation on the efficacy of lopinavir/ritonavir and chloroquine to treat nonsevere COVID19 patients. J Acquir Immune Defic Syndr. 2020;85: 239-43.

155. Abd-Elsalam S, Esmail ES, Khalaf M, et al. Hydroxychloroquine in the treatment of COVID-19: a multicenter randomized controlled study. Am J Trop Med Hyg. 2020;103:1635-9.
156. RECOVERY Collaborative Group. Effect of hydroxychloroquine in hospitalized patients with Covid19. N Engl J Med. 2020;383:2030-40.

157. Brown SM, Peltan I, Kumar N, et al. Hydroxychloroquine vs. azithromycin for hospitalized patients with COVID-19 (HAHPS): results of a randomized, active comparator trial. Ann Am Thorac Soc. 2020;18:590-7.

158. Dabbous HM, El-Sayed MH, Assal GE, et al. Safety and efficacy of favipiravir versus hydroxychloroquine in management of COVID-19: a randomised controlled trial. Sci Rep. 2021;11:7282.

159. Dubée V, Roy PM, Vielle B, et al. Hydroxychloroquine in mild-to-moderate coronavirus disease 2019: a placebo-controlled double blind trial. Clin Microbiol Infect. 2021;2021: S1198-743X(21)00140-3. https://doi.org/10.1016/j. cmi.2021.03.005.

160. Kamran SM, Moeed HA, Mirza Z, et al. Clearing the fog: is hydroxychloroquine effective in reducing coronavirus disease-2019 progression? A randomized controlled trial. Cureus. 2021;13:e14186.

161. Galan LEB, Santos NMD, Asato MS, et al. Phase 2 randomized study on chloroquine, hydroxychloroquine or ivermectin in hospitalized patients with severe manifestations of SARS-CoV-2 infection. Pathog Glob Health. 2021. https://doi.org/10.1080/ 20477724.2021.1890887.

162. Self WH, Semler MW, Leither LM, et al. Effect of hydroxychloroquine on clinical status at 14 days in hospitalized patients with COVID-19: a randomized clinical trial. JAMA. 2020;324:2165-76.

163. Ulrich RJ, Troxel AB, Carmody E, et al. Treating COVID-19 with hydroxychloroquine (TEACH): a multicenter, double-blind randomized controlled trial in hospitalized patients. Open Forum Infect Dis. 2020;7:ofaa446.

164. Kashour Z, Riaz M, Garbati MA, et al. Efficacy of chloroquine or hydroxychloroquine in COVID-19 patients: a systematic review and meta-analysis. J Antimicrob Chemother. 2021;76:30-42.

165. Fiolet T, Guihur A, Rebeaud ME, et al. Effect of hydroxychloroquine with or without azithromycin on the mortality of coronavirus disease 2019 (COVID-19) patients: a systematic review and metaanalysis. Clin Microbiol Infect. 2021;27:19-27.

166. Lyngbakken MN, Berdal JE, Eskesen A, et al. A pragmatic randomized controlled trial reports lack of efficacy of hydroxychloroquine on coronavirus disease 2019 viral kinetics. Nat Commun. 2020;11: 5284 . 
167. Tang W, Cao Z, Han M, et al. Hydroxychloroquine in patients with mainly mild to moderate coronavirus disease 2019: open label, randomised controlled trial. BMJ. 2020;369:m1849.

168. Raoult D. Rational for meta-analysis and randomized treatment: the COVID-19 example. Clin Microbiol Infect. 2021;27:6-8.

169. Lansbury L, Lim B, Baskaran V, Lim WS. Co-infections in people with COVID-19: a systematic review and meta-analysis. J Infect. 2020;81:266-75.

170. Rawson TM, Moore LSP, Zhu N, et al. Bacterial and fungal coinfection in individuals with coronavirus: a rapid review to support COVID-19 antimicrobial prescribing. Clin Infect Dis. 2020;71:2459-68.

171. Furtado RHM, Berwanger O, Fonseca HA, et al. Azithromycin in addition to standard of care versus standard of care alone in the treatment of patients admitted to the hospital with severe COVID-19 in Brazil (COALITION II): a randomised clinical trial. Lancet. 2020;396:959-67.

172. RECOVERY Collaborative Group. Azithromycin in patients admitted to hospital with COVID-19 (RECOVERY): a randomised, controlled, open-label, platform trial. Lancet. 2021;397:605-12.

173. Cao J, Tu WJ, Cheng W, et al. Clinical features and short-term outcomes of 102 patients with coronavirus disease 2019 in Wuhan, China. Clin Infect Dis. 2020;71:748-55.

174. Chen T, Wu D, Chen $\mathrm{H}$, et al. Clinical characteristics of 113 deceased patients with coronavirus disease 2019: retrospective study. BMJ. 2020;368: m1091.

175. Deng Y, Liu W, Liu K, et al. Clinical characteristics of fatal and recovered cases of coronavirus disease 2019 in Wuhan, China: a retrospective study. Chin Med J (Engl). 2020;133:1261-7.

176. Guan WJ, Ni ZY, Hu Y, et al. Clinical characteristics of coronavirus disease 2019 in China. N Engl J Med. 2020;382:1708-20.

177. Vena A, Giacobbe DR, Di Biagio A, et al. Clinical characteristics, management and in-hospital mortality of patients with coronavirus disease 2019 in Genoa. Italy Clin Microbiol Infect. 2020;26: 1537-44.

178. Wu C, Chen X, Cai Y, et al. Risk factors associated with acute respiratory distress syndrome and death in patients with coronavirus disease 2019 pneumonia in Wuhan. China JAMA Intern Med. 2020;180:934-43.
179. Zhou F, Yu T, Du R, et al. Clinical course and risk factors for mortality of adult inpatients with COVID-19 in Wuhan, China: a retrospective cohort study. Lancet. 2020;395:1054-62.

180. Cui N, Yan R, Qin C, Zhao J. Clinical characteristics and immune responses of 137 deceased patients with COVID-19: a retrospective study. Front Cell Infect Microbiol. 2020;10:595333.

181. Gutierrez-Abejon E, Tamayo E, Martin-Garcia D, et al. Clinical profile, treatment and predictors during the first COVID-19 wave: a population-based registry analysis from Castile and Leon Hospitals. Int J Environ Res Public Health. 202017(24):9360. https://doi.org/10.3390/ijerph17249360.

182. Marcolino MS, Ziegelmann PK, Souza-Silva MVR, et al. Clinical characteristics and outcomes of patients hospitalized with COVID-19 in Brazil: results from the Brazilian COVID-19 Registry. Int J Infect Dis. 2021;107:300-310.

183. Merugu GP, Neishwat Z, Balla M, et al. Predictors of mortality in 217 COVID-19 patients in Northwest Ohio, United States: a retrospective study. J Med Virol. 2021;93(5):2875-82.

184. Wang Z, Wang Z. Identification of risk factors for inhospital death of COVID-19 pneumonia-lessions from the early outbreak. BMC Infect Dis. 2021;21: 113.

185. Zhang XB, Hu L, Ming $\mathrm{Q}$, et al. Risk factors for mortality of coronavirus disease-2019 (COVID-19) patients in two centers of Hubei province, China: a retrospective analysis. PLoS One. 2021;16: e0246030.

186. Zhong J, Tang J, Ye C, Dong L. The immunology of COVID-19: is immune modulation an option for treatment? Lancet Rheumatol. 2020;2:e428-36.

187. Moore JB, June $\mathrm{CH}$. Cytokine release syndrome in severe COVID-19. Science. 2020;368:473-4.

188. Bassetti M, Ansaldi F, Icardi G, et al. COVID19:some clinical questions after the first 4 months. Eur J Clin Invest. 2020;50:e13326.

189. Xu X, Han M, Li T, et al. Effective treatment of severe COVID-19 patients with tocilizumab. Proc Natl Acad Sci USA. 2020;117:10970-5.

190. Quartuccio L, Sonaglia A, McGonagle D, et al. Profiling COVID-19 pneumonia progressing into the cytokine storm syndrome: results from a single Italian Centre study on tocilizumab versus standard of care. J Clin Virol. 2020;129:104444. 
191. Luo P, Liu Y, Qiu L, et al. Tocilizumab treatment in COVID-19: a single center experience. J Med Virol. 2020;92:814-8.

192. Dimopoulos G, de Mast Q, Markou N, et al. Favorable anakinra responses in severe covid-19 patients with secondary hemophagocytic lymphohistiocytosis. Cell Host Microbe. 2020;28:117-123e1.

193. ACTIV-3/TICO LY-CoV555 Study Group, Lundgren JD, Grund B, et al. A neutralizing monoclonal antibody for hospitalized patients with Covid-19. N Engl J Med. 2021;384(10):905-914.

194. Regeneron ${ }^{\circledR}$. REGN-COV2 independent data monitoring committee recommend holding enrollment in hospitalized patients with high oxygen requirements and continuing enrollment in patients with low or no oxygen requirements. 30 October 2020 . 2021. https://investor.regeneron.com/news-releases/ news-release-details/regn-cov2-independent-datamonitoring-committee-recommends. Accessed 10 Jan 2021.

195. Campochiaro C, Della-Torre E, Cavalli G, et al. Efficacy and safety of tocilizumab in severe COVID19 patients: a single-centre retrospective cohort study. Eur J Intern Med. 2020;76:43-9.

196. Capra R, De Rossi N, Mattioli F, et al. Impact of low dose tocilizumab on mortality rate in patients with COVID-19 related pneumonia. Eur J Intern Med. 2020;76:31-5.

197. Colaneri M, Bogliolo L, Valsecchi P, et al. Tocilizumab for treatment of severe COVID-19 patients: preliminary results from SMAtteo COvid19 REgistry (SMACORE). Microorganisms. 2020;8(5):695.

198. Cortegiani A, Ippolito M, Greco M, et al. Rationale and evidence on the use of tocilizumab in COVID19: a systematic review. Pulmonology. 2021;27: 52-66.

199. Kewan T, Covut F, Al-Jaghbeer MJ, et al. Tocilizumab for treatment of patients with severe COVID19: a retrospective cohort study. EClinicalMedicine. 2020;24:100418.

200. Martinez-Sanz J, Muriel A, Ron R, et al. Effects of tocilizumab on mortality in hospitalized patients with COVID-19: a multicentre cohort study. Clin Microbiol Infect. 2021;27(2):238-43.

201. Mikulska M, Nicolini LA, Signori A, et al. Tocilizumab and steroid treatment in patients with COVID19 pneumonia. PLoS One. 2020;15(8):e0237831.

202. Hermine O, Mariette X, Tharaux PL, et al. Effect of tocilizumab vs usual care in adults hospitalized with COVID-19 and moderate or severe pneumonia: a randomized clinical trial. JAMA Intern Med. 2021;181:32-40.

203. Salama C, Han J, Yau L, et al. Tocilizumab in patients hospitalized with Covid-19 pneumonia. N Engl J Med. 2021;384:20-30.

204. Salvarani C, Dolci G, Massari M, et al. Effect of tocilizumab vs standard care on clinical worsening in patients hospitalized with COVID-19 pneumonia: a randomized clinical trial. JAMA Intern Med. 2021;181:24-31.

205. Stone JH, Frigault MJ, Serling-Boyd NJ, et al. Efficacy of tocilizumab in patients hospitalized with Covid19. N Engl J Med. 2020;383:2333-44.

206. Rosas IO, Brau N, Waters M, et al. Tocilizumab in hospitalized patients with severe Covid-19 pneumonia. N Engl J Med. 2021;384(16):1503-1516.

207. Soin AS, Kumar K, Choudhary NS, et al. Tocilizumab plus standard care versus standard care in patients in India with moderate to severe COVID19 -associated cytokine release syndrome (COVINTOC): an open-label, multicentre, randomised, controlled, phase 3 trial. Lancet Respir Med. 2021;9: 511-21.

208. Veiga VC, Prats JAGG, Farias DLC, et al. Effect of tocilizumab on clinical outcomes at 15 days in patients with severe or critical coronavirus disease 2019: randomised controlled trial. BMJ. 2021;372: n84.

209. Parr JB. Time to reassess tocilizumab's role in COVID-19 pneumonia. JAMA Intern Med. 2021;181:12-5.

210. RECOVERY Collaborative Group. Tocilizumab in patients admitted to hospital with COVID-19 (RECOVERY): a randomised, controlled, open-label, platform trial. Lancet. 2021;397:1637-45.

211. REMAP-CAP Investigators. Interleukin-6 receptor antagonists in critically ill patients with Covid-19. N Engl J Med. 2021;384(16):1491-1502.

212. Lescure FX, Honda H, Fowler RA, et al. Sarilumab in patients admitted to hospital with severe or critical COVID-19: a randomised, double-blind, placebocontrolled, phase 3 trial. Lancet Respir Med. 2021;9: 522-32.

213. CORIMUNO-19 Collaborative group. Effect of anakinra versus usual care in adults in hospital with COVID-19 and mild-to-moderate pneumonia (CORIMUNO-ANA-1): a randomised controlled trial. Lancet Respir Med. 2021;9(3):295-304. 
214. Kalil AC, Patterson TF, Mehta AK, et al. Baricitinib plus remdesivir for hospitalized adults with Covid19. N Engl J Med. 2021;384(9):795-807.

215. Goletti D, Cantini F. Baricitinib therapy in Covid-19 pneumonia-an unmet need fulfilled. N Engl J Med. 2021;384:867-9.

216. Lopes MI, Bonjorno LP, Giannini MC, et al. Beneficial effects of colchicine for moderate to severe COVID-19: a randomised, double-blinded, placebocontrolled clinical trial. RMD Open. 2021;7: e001455.

217. Horby PW, Campbell M, Spata E, et al. Colchicine in patients admitted to hospital with COVID-19 (RECOVERY): a randomised, controlled, open-label, platform trial. medRxiv. 2021.05.18.21257267. https://doi.org/10.1101/2021.05.18.21257267.

218. Estcourt LJ, Roberts DJ. Convalescent plasma for covid-19. BMJ. 2020;370:m3516.

219. Wood EM, Estcourt LJ, McQuilten Z. How should we use convalescent plasma therapies for COVID19? Blood. 2021;137(12):1573-81.

220. Ahn JY, Sohn Y, Lee SH, et al. Use of convalescent plasma therapy in two COVID-19 patients with acute respiratory distress syndrome in Korea. J Korean Med Sci. 2020;35:e149.

221. Anderson J, Schauer J, Bryant S, Graves CR. The use of convalescent plasma therapy and remdesivir in the successful management of a critically ill obstetric patient with novel coronavirus 2019 infection: a case report. Case Rep Womens Health. 2020;27:e00221.

222. Cinar OE, Sayinalp B, Aladag Karakulak E, et al. Convalescent (immune) plasma treatment in a myelodysplastic COVID-19 patient with disseminated tuberculosis. Transfus Apher Sci. 2020;59: 102821.

223. Duan K, Liu B, Li C, et al. Effectiveness of convalescent plasma therapy in severe COVID-19 patients. Proc Natl Acad Sci USA. 2020;117:9490-6.

224. Ibrahim D, Dulipsingh L, Zapatka L, et al. Factors associated with good patient outcomes following convalescent plasma in COVID-19: a prospective phase II clinical trial. Infect Dis Ther. 2020;9(4): 913-26.

225. Joyner MJ, Bruno KA, Klassen SA, et al. Safety update: COVID-19 convalescent plasma in 20,000 hospitalized patients. Mayo Clin Proc. 2020;95: 1888-97.
226. Joyner MJ, Wright RS, Fairweather D, et al. Early safety indicators of COVID-19 convalescent plasma in 5000 patients. J Clin Invest. 2020;130:4791-7.

227. Kong Y, Cai C, Ling L, et al. Successful treatment of a centenarian with coronavirus disease 2019 (COVID-19) using convalescent plasma. Transfus Apher Sci. 2020;59:102820.

228. Liu STH, Lin HM, Baine I, et al. Convalescent plasma treatment of severe COVID-19: a propensity score-matched control study. Nat Med. 2020;26: 1708-13.

229. Salazar E, Christensen PA, Graviss EA, et al. Treatment of coronavirus disease 2019 patients with convalescent plasma reveals a signal of significantly decreased mortality. Am J Pathol. 2020;190: 2290-303.

230. Salazar E, Perez KK, Ashraf M, et al. Treatment of coronavirus disease 2019 (COVID-19) patients with convalescent plasma. Am J Pathol. 2020;190: 1680-90.

231. Shen C, Wang Z, Zhao F, et al. Treatment of 5 critically ill patients with COVID-19 with convalescent plasma. JAMA. 2020;323:1582-9.

232. Ye M, Fu D, Ren Y, et al. Treatment with convalescent plasma for COVID-19 patients in Wuhan. China J Med Virol. 2020;92:1890-901.

233. Zeng QL, Yu ZJ, Gou JJ, et al. Effect of convalescent plasma therapy on viral shedding and survival in patients with coronavirus disease 2019. J Infect Dis. 2020;222:38-43.

234. Zhang B, Liu S, Tan T, et al. Treatment with convalescent plasma for critically ill patients with severe acute respiratory syndrome coronavirus 2 infection. Chest. 2020;158:e9-13.

235. Zhang L, Pang R, Xue X, et al. Anti-SARS-CoV-2 virus antibody levels in convalescent plasma of six donors who have recovered from COVID-19. Aging (Albany NY). 2020;12:6536-42.

236. Perotti C, Baldanti F, Bruno R, et al. Mortality reduction in 46 severe Covid-19 patients treated with hyperimmune plasma. A proof of concept single arm multicenter trial. Haematologica. 2020;105:2834-2840.

237. Agarwal A, Mukherjee A, Kumar G, et al. Convalescent plasma in the management of moderate covid-19 in adults in India: open label phase II multicentre randomised controlled trial (PLACID Trial). BMJ. 2020;371:m3939.

238. Li L, Zhang W, Hu Y, et al. Effect of convalescent plasma therapy on time to clinical improvement in 
patients with severe and life-threatening COVID19: a randomized clinical trial. JAMA. 2020;324: 460-70.

239. Simonovich VA, Burgos Pratx LD, Scibona P, et al. A randomized trial of convalescent plasma in Covid19 severe pneumonia. N Engl J Med. 2021;384(7): 619-29.

240. Libster R, Perez Marc G, Wappner D, et al. Early high-titer plasma therapy to prevent severe Covid19 in older adults. N Engl J Med. 2021;384(7): 610-18.

241. AlQahtani M, Abdulrahman A, Almadani A, et al. Randomized controlled trial of convalescent plasma therapy against standard therapy in patients with severe COVID-19 disease. Sci Rep. 2021;11:9927.

242. RECOVERY Collaborative Group. Convalescent plasma in patients admitted to hospital with COVID-19 (RECOVERY): a randomised controlled, open-label, platform trial. Lancet. 2021;S0140-6736(21):00897-907.

243. Bajpai M, Kumar S, Maheshwari A, et al. Efficacy of convalescent plasma therapy compared to fresh frozen plasma in severely ill COVID-19 patients: a pilot randomized controlled trial. medRxiv. 2020;2020.2010.2025.20219337.

244. Balcells ME, Rojas L, Le Corre N, et al. Early antiSARS-CoV-2 convalescent plasma in patients admitted for COVID-19: a randomized phase II clinical trial. medRxiv. 2020;2020.2009.2017. 20196212.

245. Gharbharan A, Jordans CCE, Geurtsvankessel C, et al. Convalescent plasma for COVID-19. a randomized clinical trial. medRxiv. 2020;2020.2007. 2001.20139857.

246. Ray Y, Paul SR, Bandopadhyay P, et al. Clinical and immunological benefits of convalescent plasma therapy in severe COVID-19: insights from a single center open label randomised control trial. medRxiv. 2020;2020.2011.2025.20237883.

247. O'Donnell MR, Grinsztejn B, Cummings MJ, et al. A randomized, double-blind, controlled trial of convalescent plasma in adults with severe COVID-19. medRxiv. 2021; 2021.03.12.21253373. https://doi. org/10.1101/2021.03.12.21253373.

248. Avendano-Sola C, Ramos-Martinez A, Munez-Rubio $\mathrm{E}$, et al. Convalescent plasma for COVID-19: a multicenter, randomized clinical trial. medRxiv. 2020;2020:2020.2008.2026.20182444.

249. Katz LM. (A little) clarity on convalescent plasma for Covid-19. N Engl J Med. 2021;384(7):666-8.
250. Grieco DL, Menga LS, Cesarano M, et al. Effect of helmet noninvasive ventilation vs high-flow nasal oxygen on days free of respiratory support in patients with COVID-19 and moderate to severe hypoxemic respiratory failure: the HENIVOT randomized clinical trial. JAMA. 2021;325:1731-43.

251. Munshi L, Hall JB. Respiratory support during the COVID-19 pandemic: is it time to consider using a helmet? JAMA. 2021;325:1723-5.

252. Aliberti S, Radovanovic D, Billi F, et al. Helmet CPAP treatment in patients with COVID-19 pneumonia: a multicentre cohort study. Eur Respir J. 2020;56(4):2001935.

253. Alviset S, Riller Q, Aboab J, et al. Continuous positive airway pressure (CPAP) face-mask ventilation is an easy and cheap option to manage a massive influx of patients presenting acute respiratory failure during the SARS-CoV-2 outbreak: a retrospective cohort study. PLoS One. 2020;15:e0240645.

254. Arina P, Baso B, Moro V, et al. Discriminating between CPAP success and failure in COVID-19 patients with severe respiratory failure. Intensive Care Med. 2021;47(2):237-9.

255. Avdeev SN, Yaroshetskiy AI, Tsareva NA, et al. Noninvasive ventilation for acute hypoxemic respiratory failure in patients with COVID-19. Am J Emerg Med. 2021;39:154-7.

256. Brusasco C, Corradi F, Di Domenico A, et al. Continuous positive airway pressure in Covid-19 patients with moderate-to-severe respiratory failure. Eur Respir J. 2021;57:2002524.

257. Burns GP, Lane ND, Tedd HM, et al. Improved survival following ward-based non-invasive pressure support for severe hypoxia in a cohort of frail patients with COVID-19: retrospective analysis from a UK teaching hospital. BMJ Open Respir Res. 2020;;7(1):e000621.

258. Duca A, Memaj I, Zanardi F, et al. Severity of respiratory failure and outcome of patients needing a ventilatory support in the emergency department during Italian novel coronavirus SARS-CoV2 outbreak: preliminary data on the role of helmet CPAP and non-invasive positive pressure ventilation. EClinicalMedicine. 2020;24:100419.

259. Franco C, Facciolongo N, Tonelli R, et al. Feasibility and clinical impact of out-of-ICU noninvasive respiratory support in patients with COVID-19-related pneumonia. Eur Respir J. 2020;56(5):2002130.

260. Hallifax RJ, Porter BM, Elder PJ, et al. Successful awake proning is associated with improved clinical outcomes in patients with COVID-19: single-centre 
high-dependency unit experience. BMJ Open Respir Res. 2020;7(1):e000678.

261. Karagiannidis C, Mostert C, Hentschker C, et al. Case characteristics, resource use, and outcomes of 10021 patients with COVID-19 admitted to 920 German hospitals: an observational study. Lancet Respir Med. 2020;8:853-62.

262. Knights H, Mayor N, Millar K, et al. Characteristics and outcomes of patients with COVID-19 at a district general hospital in Surrey. UK Clin Med (Lond). 2020;20:e148-53.

263. Mukhtar A, Lotfy A, Hasanin A, et al. Outcome of non-invasive ventilation in COVID-19 critically ill patients: a retrospective observational study. Anaesth Crit Care Pain Med. 2020;39:579-80.

264. Nightingale R, Nwosu N, Kutubudin F, et al. Is continuous positive airway pressure (CPAP) a new standard of care for type 1 respiratory failure in COVID-19 patients? A retrospective observational study of a dedicated COVID-19 CPAP service. BMJ Open Respir Res. 2020;7(1):e000639.

265. Noeman-Ahmed Y, Gokaraju S, Powrie DJ, et al. Predictors of CPAP outcome in hospitalized COVID19 patients. Respirology. 2020;25:1316-9.

266. Oranger M, Gonzalez-Bermejo J, Dacosta-Noble P, et al. Continuous positive airway pressure to avoid intubation in SARS-CoV-2 pneumonia: a two-period retrospective case-control study. Eur Respir J. 2020;56(2):2001692.

267. Rahim F, Amin S, Noor M, et al. Mortality of patients with severe COVID-19 in the intensive care unit: an observational study from a major COVID19 receiving hospital. Cureus. 2020;12:e10906.

268. Ramirez GA, Bozzolo EP, Castelli E, et al. Continuous positive airway pressure and pronation outside the intensive care unit in COVID 19 ARDS. Minerva Med. 2020. https://doi.org/10.23736/S0026-4806. 20.06952-9.

269. Sivaloganathan AA, Nasim-Mohi M, Brown MM, et al. Noninvasive ventilation for COVID-19-associated acute hypoxaemic respiratory failure: experience from a single centre. Br J Anaesth. 2020;125: e368-71.

270. Bellani G, Grasselli G, Cecconi M, et al. Noninvasive ventilatory support of COVID-19 patients outside the intensive care units (WARd-COVID). Ann Am Thorac Soc. 2021;18(6):1020-26.

271. Potalivo A, Montomoli J, Facondini F, et al. Sixtyday mortality among 520 Italian hospitalized COVID-19 patients according to the adopted ventilatory strategy in the context of an integrated multidisciplinary clinical organization: a population-based cohort study. Clin Epidemiol. 2020;12: 1421-31.

272. Carpagnano GE, Buonamico E, Migliore G, et al. Bilevel and continuous positive airway pressure and factors linked to all-cause mortality in COVID-19 patients in an intermediate respiratory intensive care unit in Italy. Expert Rev Respir Med. 2020;15(6):853-7.

273. De Vita N, Scotti L, Cammarota G, et al. Predictors of intubation in COVID-19 patients treated with out-of-ICU continuous positive airway pressure. Pulmonology. 2021. https://doi.org/10.1016/j. pulmoe.2020.12.010.

274. Faraone A, Beltrame C, Crociani A, et al. Effectiveness and safety of noninvasive positive pressure ventilation in the treatment of COVID-19-associated acute hypoxemic respiratory failure: a single center, non-ICU setting experience. Intern Emerg Med. 2020. https://doi.org/10.1007/s11739-02002562-2

275. Thompson JV, Meghani NJ, Powell BM, et al. Patient characteristics and predictors of mortality in 470 adults admitted to a district general hospital in England with Covid-19. Epidemiol Infect. 2020;148: e285.

276. Walker J, Dolly S, Ng L, et al. The role of CPAP as a potential bridge to invasive ventilation and as a ceiling-of-care for patients hospitalized with Covid19-an observational study. PLoS One. 2020;15: e0244857.

277. Centers for Disease Control and Prevention. Discontinuation of transmission-based precautions and disposition of patients with COVID-19 in healthcare settings (interim guidance). Version of 16 Feb 2021. 2021. https://www.cdc.gov/ coronavirus/2019-ncov/hcp/dispositionhospitalized-patients.html. Accessed 17 May 2021.

278. European Centers for Disease Control and Prevention. Rapid risk assessment. Increased transmission of COVID-19 in the EU/EEA and the UK-thirteenth update. Version of 23 October 2020. 2020. https:// www.ecdc.europa.eu/sites/default/files/documents/ RRA-COVID-19-EU-EEA-UK-thirteenth-update-23Oct-2020.pdf. Accessed 17 Jan 2020.

279. Ministero della Salute. Covid-19: indicazioni per la durata e il termine dell'isolamento e della quarantena. Circolare del ministero della Salute del 12 ottobre 2020. 2020. https://www.trovanorme. salute.gov.it/norme/renderNormsanPdf?anno= $2020 \&$ codLeg $=76613 \&$ parte $=1 \% 20 \&$ serie $=$ null. Accessed 27 May 2021. 